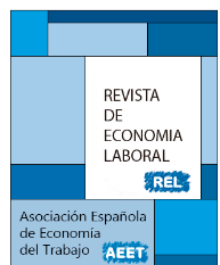

\title{
REFORMA LABORAL, DEVALUACIÓN SALARIAL Y EMPLEO: UNA PERSPECTIVA MACROECONÓMICA
}

\author{
Jorge Uxó1, Eladio Febrero, Fernando Bermejo \\ Universidad de Castilla-La Mancha
}

Recibido Julio 2015; Aceptado Octubre 2015

\section{Resumen}

El objetivo de este artículo es analizar la reforma laboral aprobada en 2012 desde una perspectiva macroeconómica, en el sentido de que nos interesa comprender su inserción en el contexto global de la estrategia de politica económica que han aplicado las autoridades, y su impacto en el desempleo registrado por la economía española. Se ocupa principalmente del efecto que la reforma laboral ha tenido en el comportamiento agregado de los salarios, y su influencia, junto a otros elementos con los que se combina, como la austeridad fiscal, en la evolución del desempleo. Nuestra conclusión es que la caída de los salarios impulsada por la reforma laboral ha sido perjudicial para el empleo porque el efecto restrictivo sobre la demanda interna -que se suma al que se deriva de la austeridad fiscal- ha sido mayor que el impulso sobre la demanda externa.

Palabras clave: reforma laboral, devaluación interna, salarios, competitividad. Clasificación JEL: E63, E64, E65.

\section{Abstract}

The main objective of this article is to analyse the labour market reform of 2012 from a macroeconomic perspective, with an especial focus on the role played by this reform in the economic policy strategy developed by the authorities and its impact on the performance of the Spanish economy. Specifically, we review the effect of the reform on the aggregate behavior of wages, and its influence-jointly with the other elements that conform that strategy, as fiscal austerity-on employment. Our main conclusion is that the decrease in wages provoked by the labour market reform has been detrimental for employment, because its restrictive effect on domestic demand-added to fiscal austerityhas not been offset by an impulse on external demand.

Key words: Labour market reform, Internal devaluation, Wages, Competitiveness.

JEL Classification: E63, E64, E65.

1 Agradecemos las sugerencias y comentarios de un evaluador anónimo y de los asistentes al Workshop "Internal devaluation and employment in Europe", celebrado en el European Trade Union Institute (Bruselas, enero 2015) donde presentamos una versión previa de este trabajo. Autor para correspondencia: jorge.uxo@uclm.es

C Revista de Economía Laboral 


\section{Introducción}

El objetivo de este artículo es analizar la reforma laboral aprobada en 2012 desde una perspectiva macroeconómica, en el sentido de que nos interesa comprender su inserción en el contexto global de la estrategia de política económica que han aplicado las autoridades, y su impacto en el desempleo registrado por la economía española.

Concretando más, vamos a ocuparnos principalmente del efecto que la reforma laboral ha tenido en el comportamiento agregado de los salarios, y su influencia, junto a otros elementos con los que se combina, como la austeridad fiscal, en la evolución del desempleo.

En un estudio sobre el diferente comportamiento del paro en distintos países europeos desde el inicio de la Gran Recesión, Myant y Piasna (2014) señalan dos posibles explicaciones alternativas de las diferencias que se observan. Una de ellas está centrada en el funcionamiento del mercado de trabajo, y atribuye el mayor impacto sobre el empleo de la caída en el PIB que experimentan algunos países a la "rigidez" de sus mercados de trabajo, entendido esto en un sentido amplio (por ejemplo, dificultades para hacer cambios en las condiciones de trabajo, jornadas laborales y salarios). La segunda explicación se centra más bien en factores de carácter estructural, y sobre todo en el tipo de perturbación que experimenta cada país y su especialización productiva. Desde este punto de vista, para explicar el mayor aumento del paro en algunos países sería más relevante el peso que tenía antes de la crisis el sector de la construcción, o el pinchazo de una burbuja crediticia e inmobiliaria, que la falta de flexibilidad salarial.

Para verificar la importancia de ambas hipótesis, lo que estos autores proponen es, en primer lugar, comprobar si el aumento del desempleo que se ha producido en el país analizado puede asociarse o no con un shock específico en un sector, como puede ser el de la construcción. En segundo lugar, para verificar la importancia de la hipótesis de la rigidez salarial, ver los efectos sobre el empleo de las reformas laborales aplicadas después de la crisis con el fin de aumentar la flexibilidad a la baja de los salarios. Si, una vez aplicadas estas reformas, los salarios bajan, pero el desempleo no se reduce o incluso aumenta, la explicación basada en la rigidez salarial perdería una parte importante de su capacidad para explicar el mayor aumento del paro en algunos países. Nosotros tomamos este esquema como punto de partida para nuestro análisis y lo aplicamos al caso de España y a los efectos de la reforma del mercado de trabajo. 
Debemos aceptar que esta forma de evaluar la influencia de la reforma laboral sobre el empleo se enfrenta al problema de que esta medida no se aplica en un contexto en el que el resto de factores que lo afectan se mantienen invariables. Sin embargo, creemos que en el caso de España la reforma del mercado de trabajo se inserta en una estrategia global de política económica que combina la devaluación interna con la austeridad fiscal, y que por ello mismo es coherente abordar conjuntamente sus efectos sobre el empleo. No es que, por ejemplo, los efectos beneficiosos de la reforma laboral no se hayan podido manifestar porque, simultáneamente, la política fiscal era restrictiva, sino que ambas piezas forman parte de la misma orientación política, cuyos efectos netos sobre el empleo se pretenden positivos. Nosotros queremos contrastar si ha sido así o no.

El artículo se organiza de la siguiente forma. En el próximo apartado resumimos algunos aspectos de la evolución del empleo y el paro que nos interesa destacar, así como las principales características de los desempleados. En el tercer apartado presentamos evidencia sobre la composición sectorial del empleo, para destacar la importancia fundamental que ha tenido la caída de la actividad de la construcción. El cuarto apartado está dedicado a los efectos sobre los salarios y el empleo de la reforma laboral, entendiendo que ésta es una pieza fundamental de la estrategia de política macroeconómica aplicada en España en estos años. Por último, ofrecemos algunas conclusiones.

\section{Algunas características de la evolución del empleo y el desempleo en España}

Nuestro periodo de análisis se refiere a los años 2008 a 2014, y los gráficos 1 y 2 comparan la evolución temporal del empleo y el PIB en España y en la UE28 durante este tiempo. A finales de 2014 se habían destruido en España 3,3 millones de empleos, o el 16\% del empleo total que había en el segundo trimestre de 2008 (inmediatamente antes de que empezaran a registrarse tasas de crecimiento negativas). En ese mismo periodo, el empleo sólo se redujo un $2 \%$ en la UE28. Es decir, que España ha tenido un comportamiento relativo del empleo mucho peor durante estos años.

Por otra parte, en el gráfico 2 hacemos la misma comparación, pero considerando ahora la evolución del PIB real. Y una conclusión inmediata que obtenemos es que al menos una parte importante de esa mayor destrucción del empleo tiene que ver con una caída más acusada del PIB, que en la UE ha vuelto a su nivel inicial de 2008, mientras que 
en España es aún un 6\% inferior. Sin embargo, conviene distinguir varios periodos.

El primero se refiere a los años 2008-2009, en los que la caída del PIB no fue superior en España que en la UE, pero el empleo sí se redujo mucho más. Esto significa que la elasticidad del empleo respecto a la actividad económica fue significativamente más elevada en España durante la Gran Recesión (de hecho, esta es una característica del empleo en España que se ha manifestado también en otros periodos).

El segundo periodo (2010-2013) coincide con el giro hacia una política de austeridad fiscal y con la aplicación de las distintas reformas laborales posteriores a la crisis (en 2010, 2011 y, sobre todo, 2012). Lo relevante en este caso para entender la diferencia en el comportamiento del empleo es que mientras en Europa el PIB estaba creciendo (un 1\% acumulado en 2011-2013) España vuelve a experimentar un periodo de recesión (el PIB se redujo un 3\%). Respecto al empleo, en España cayó un $9 \%$ y se mantuvo constante en el conjunto de la UE. En nuestra opinión, esto sitúa en primer plano las políticas macroeconómicas aplicadas en España durante este periodo, y su influencia sobre la tasa de crecimiento, como el principal factor explicativo de las diferencias en el comportamiento del empleo.

\section{Gráfico 1: Empleo (2008Q2=100)}

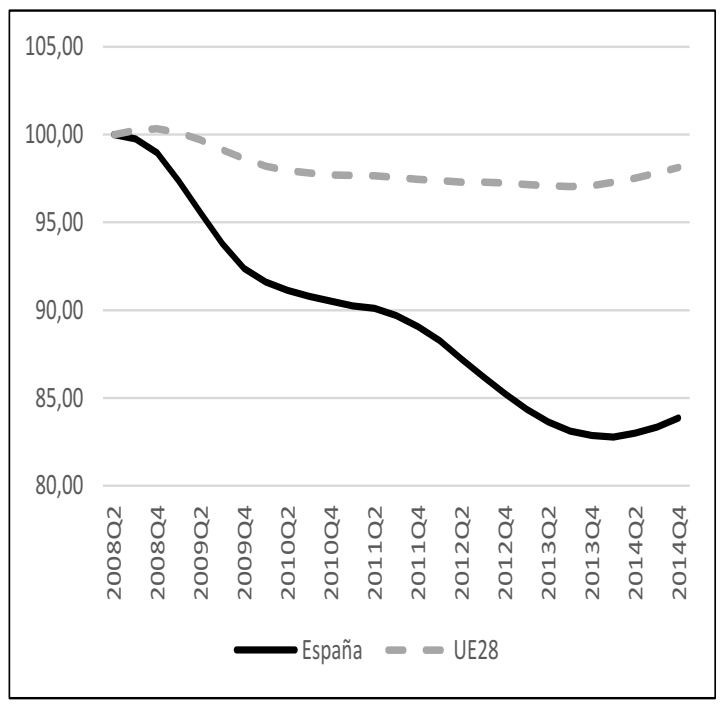

Fuente: Eurostat.

\section{Gráfico 2: PIB real (2008Q2=100)}

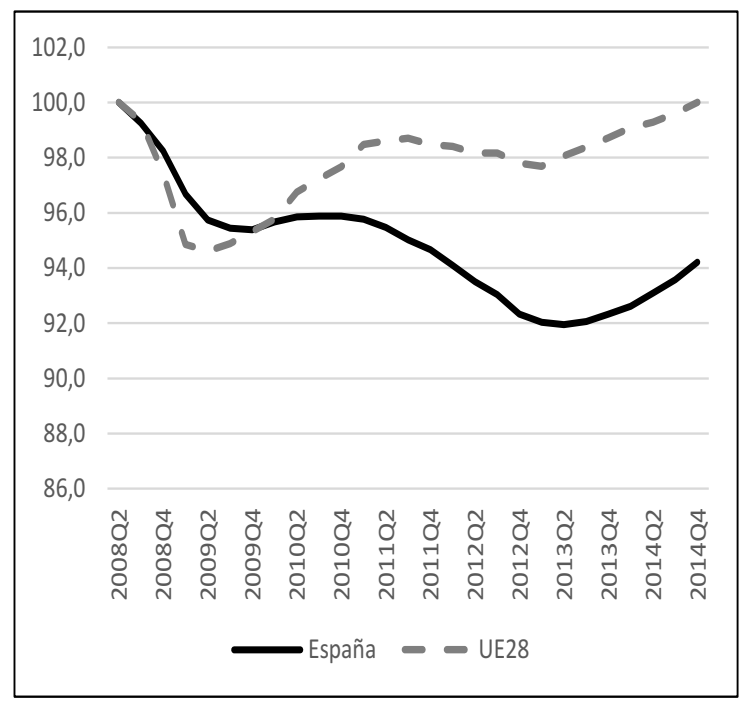


Finalmente, España ha vuelto a experimentar en 2014 un crecimiento positivo del PIB y del empleo, principalmente por el cambio de orientación en la política monetaria del BCE y en la política fiscal (la consolidación fiscal se suaviza mucho y el gasto público deja de reducirse), la depreciación del euro y la caída de los precios del petróleo.

El cuadro 1 recoge algunas características de la evolución del empleo en España y en la UE durante estos años, y otra idea que podemos destacar es que la reforma laboral no ha contribuido a eliminar la precariedad laboral, sino que la ha incrementado, especialmente entre los jóvenes (cuadro 2). Aunque la ratio de temporalidad ha decrecido en estos años desde el $30,5 \%$ al $24 \%$, lo ha hecho principalmente porque la mayor parte de la destrucción de empleo se ha producido entre los trabajadores temporales. Sin embargo, no se ha producido un aumento simultáneo de los trabajadores indefinidos, sino al contrario: se han perdido un millón de puestos de trabajo con contrato indefinido, sobre una reducción total de 2,8 millones de asalariados. El número de trabajadores a tiempo parcial ha aumentado sustancialmente a pesar de la destrucción tan grande de empleo que ha tenido lugar, y a finales de 2014 suponían ya el $16 \%$ del empleo total (4,4 puntos más que en 2008). Por tanto, las horas trabajadas se han reducido incluso más que el empleo total en estos años $(-18 \%$ frente a $-16 \%)$. Y hay un $7 \%$ de asalariados que tienen simultáneamente un contrato temporal a tiempo parcial.

De hecho, el grueso del reciente incremento en el empleo se está concretando de nuevo en contratos temporales. Entre el cuarto trimestre de 2013 y el cuarto trimestre de 2014 el número de asalariados temporales aumentó en 173.000, mientras que el número de indefinidos sólo aumentó en 43.000. De acuerdo con la EPA, además, el $14 \%$ de los empleos creados durante los tres primeros trimestres de 2014 tenía una semana laboral de menos de 10 horas, y sólo el 53\% alcanzaba las 40 horas semanales. El número medio de horas fue de 31 para los nuevos empleos, mientras que en el caso de los asalariados con más de cuatro años de antigüedad en la misma empresa la media es de 37 horas semanales. Y estas diferencias se están incrementando desde el inicio de la crisis, y especialmente a partir de la reforma laboral de 2012 .

La destrucción de empleo ha dado lugar a un aumento en el desempleo, tanto en números absolutos como en porcentaje de la población activa. La tasa de paro pasó en España del 9,1\% al 24,4\% en 2014, habiendo alcanzado el $27 \%$ en el tercer trimestre de 2013 (gráfico 3). En la UE, la tasa de paro ha pasado del 6,9\% al 10,3\%. 


\section{Cuadro 1: Principales indicadores del mercado de trabajo}

\begin{tabular}{lrrrr}
\hline \multicolumn{1}{c}{ ESPAÑA } & \multicolumn{1}{c}{$2008 \mathrm{Q} 2$} & \multicolumn{1}{c}{$2014 \mathrm{Q} 4$} & Diferencia & \% cambio \\
\hline Empleo & 20684,6 & 17344,2 & $-3340,4$ & $-16,1 \%$ \\
Tasa de empleo (\% pob>15) & $53,6 \%$ & $44,5 \%$ & $-9,1$ & \\
Asalariados indefinidos & 11858,9 & 10857,1 & $-1001,8$ & $-8,4 \%$ \\
Asalariados temporales & 5200,4 & 3428,7 & $-1771,7$ & $-34,1 \%$ \\
Tasa de temporalidad (\% & $30,5 \%$ & $24,0 \%$ & $-6,5$ & \\
Empleo a tiempo parcial & 2375,1 & 2758,8 & 383,8 & $16,2 \%$ \\
Tasa de empleo a tiempo & $11,5 \%$ & $15,9 \%$ & 4,4 & \\
Desempleados & 2081,1 & 5610,4 & 3529,3 & $169,6 \%$ \\
Tasa de desempleo & $9,1 \%$ & $24,4 \%$ & 15,3 & \\
Población mayor de 15 años & 38601,7 & 38953,3 & 351,6 & $0,9 \%$ \\
Población activa & 22765,7 & 22954,6 & 188,9 & $0,8 \%$ \\
Población inactiva & 15836,1 & 15998,7 & 162,7 & $1,0 \%$ \\
\hline \multicolumn{1}{c}{ UE28 } & $2008 \mathrm{Q} 2$ & $2014 \mathrm{Q} 3$ & Diferencia & $\%$ cambio \\
\hline Empleo & 221924,3 & 217271,5 & $-4652,8$ & $-2,1 \%$ \\
Tasa de empleo (\% pob>15) & $53,4 \%$ & $51,7 \%$ & $-1,7$ & \\
Asalariados indefinidos & 158021,0 & 156061,7 & $-1959,3$ & $-1,2 \%$ \\
Asalariados temporales & 26639,6 & 25263,7 & $-1375,9$ & $-5,2 \%$ \\
Tasa de temporalidad (\% & $14,4 \%$ & $13,9 \%$ & $-0,5$ & \\
Empleo a tiempo parcial & 40124,1 & 44412,0 & 4287,9 & $10,7 \%$ \\
Tasa de empleo a tiempo & $18,1 \%$ & $20,4 \%$ & 2,3 & \\
Desempleados & 16510,6 & 24954,7 & 8444,1 & $51,1 \%$ \\
Tasa de desempleo & $6,9 \%$ & $10,3 \%$ & 3,4 & \\
Población mayor de 15 años & 415757,2 & 420598,8 & 4841,6 & $1,2 \%$ \\
Población activa & 238434,9 & 242226,1 & 3791,2 & $1,6 \%$ \\
Población inactiva & 177322,3 & 178372,7 & 1050,4 & $0,6 \%$ \\
\hline Fuente: Eurostat & & & &
\end{tabular}

Fuente: Eurostat

Nota: Salvo que se indique otra cosa, las cifras de los cuadros recogen medias anuales en miles.

Cuadro 2: Asalariados por edad y tipo de contrato

\begin{tabular}{lcccc}
\hline & 2008 & 2010 & 2012 & 2014 \\
\hline Total asalariados & 16861,3 & 15592,3 & 14573,4 & 14285,7 \\
\% Tiempo parcial & $11,8 \%$ & $13,0 \%$ & $14,5 \%$ & $15,9 \%$ \\
\% Temporal & $29,1 \%$ & $24,7 \%$ & $23,4 \%$ & $24,0 \%$ \\
Total 15-29 & 4162,1 & 3106,8 & 2395,2 & 2139,7 \\
\% Tiempo parcial & $15,8 \%$ & $19,8 \%$ & $24,0 \%$ & $27,8 \%$ \\
\% Temporal & $48,5 \%$ & $45,1 \%$ & $47,3 \%$ & $51,9 \%$ \\
Total 30-60 & 12126,6 & 11911,3 & 11596,2 & 11530,6 \\
\% Tiempo parcial & $10,3 \%$ & $11,3 \%$ & $12,6 \%$ & $13,9 \%$ \\
\% Temporal & $23,3 \%$ & $20,1 \%$ & $19,1 \%$ & $19,7 \%$ \\
Total 60 o más & 572,6 & 574,2 & 582,0 & 615,4 \\
\% Tiempo parcial & $15,2 \%$ & $15,9 \%$ & $16,5 \%$ & $16,3 \%$ \\
\% Temporal & $11,2 \%$ & $10,6 \%$ & $10,2 \%$ & $8,2 \%$ \\
\hline
\end{tabular}

Fuente: INE 


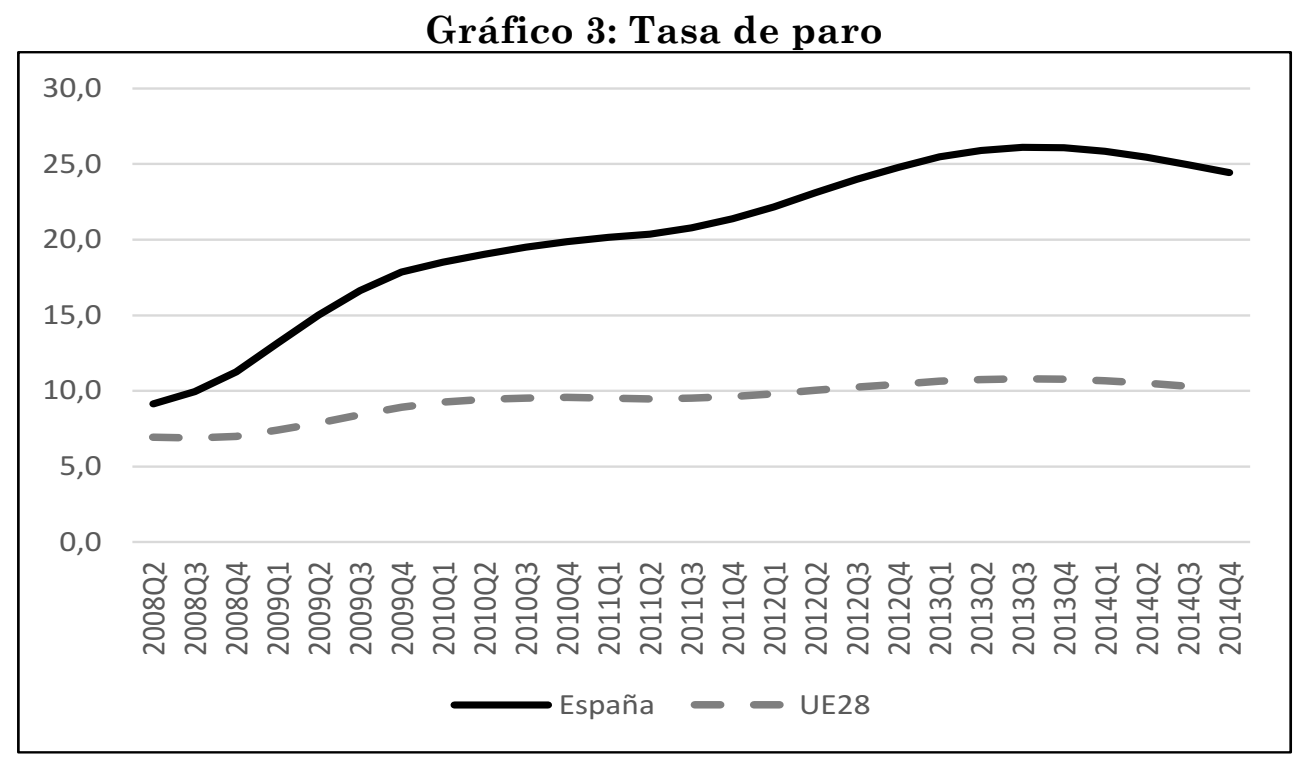

Fuente: Eurostat.

\section{Gráfico 4: Población total mayor de} 15 años

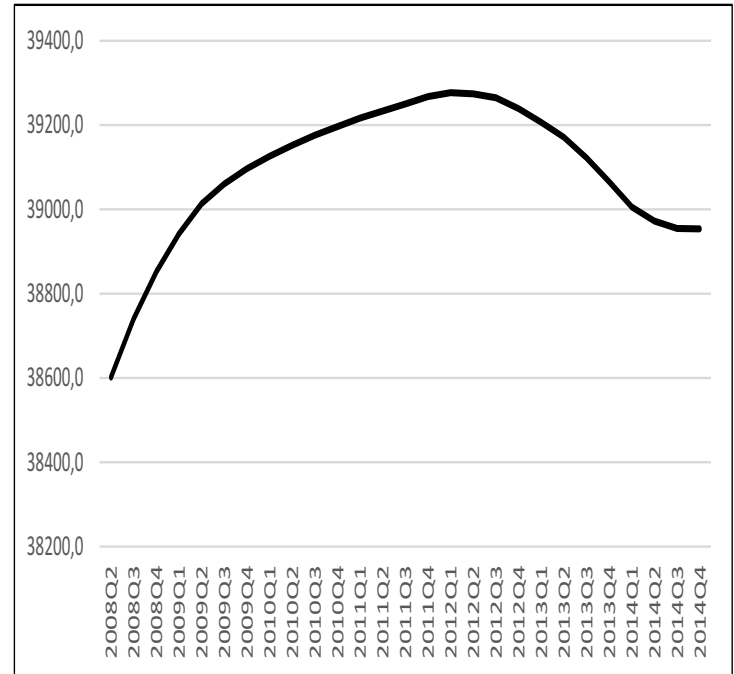

Fuente: INE.

Nota: En el gráfico 5, la población activa se mide en el eje de la derecha y la inactiva en el de la izquierda.
Gráfico 5: Población activa e inactiva

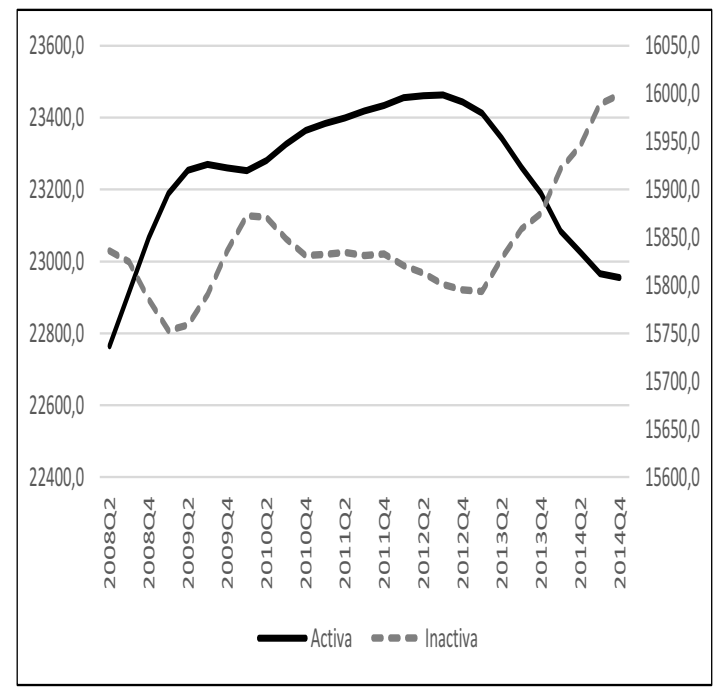


La población activa había estado creciendo rápidamente durante la anterior fase expansiva, principalmente por la llegada de inmigrantes. Entre 2009 y 2011 moderó su ritmo de crecimiento, aunque con un comportamiento muy distinto entre el caso de los hombres -decreciente- y las mujeres -creciente-. Finalmente, a partir de 2012 se ha reducido de forma sostenida. En 2014 había 489.000 activos menos que en 2012. En parte, esto se explica por la reducción de la población mayor de 15 años (gráfico 4) pero también se debe al fenómeno de los "desanimados": la población inactiva se ha incrementado en 189.000 personas (gráfico 5). En particular, el número de activos entre 16 y 29 años se ha reducido en 475.000 durante los dos últimos años. Para explicarlo, tenemos que tener en cuenta que 197.000 personas de esas edades han dejado España (20.000 eran españoles y el resto extranjeros; el flujo migratorio neto ha sido de -46.000); y que la tasa de actividad ha decrecido desde el 66\% en 2009 al 58\% en 2014.

Concluimos esta sección con una breve descripción de la distribución de los desempleados según diversos criterios. Respecto a la edad de los desempleados y su nivel de formación, el cuadro 3 muestra que la tasa de desempleo de los menores de 29 años es la más alta (para los menores de 24 la tasa de desempleo supera incluso el 50\%). El desempleo es también mucho mayor entre los individuos con bajos niveles de educación, aunque es mayor para los que han alcanzado estudios universitarios que para aquellos con un nivel de bachiller superior.

El cuadro 4 muestra la distribución del desempleo por sectores, clasificado además en función de su duración. Debe tenerse en cuenta que las cifras de los que llevan desempleados más de un año no aparecen en su sector de actividad correspondiente, sino en las columnas del bloque inferior denominado "Desempleados más de un año". Esto explica por qué las cifras de desempleo para la construcción son menores de lo esperado. En cualquier caso, el hecho más destacado corresponde a la enorme cantidad de individuos que llevan parados más de 2 años, que representan casi el $40 \%$ de los desempleados totales en 2014 (50\% si se incluyen aquellos que nunca han trabajado).

Por último, el cuadro 5 incluye los datos de desempleo clasificados en función de la ocupación que tenía el desempleado antes del despido. Según los datos de 2014, además de los desempleados de larga duración, la tasa más alta de desempleo corresponde a las ocupaciones que requieren un nivel de cualificación más bajo, concretamente, a los trabajadores dedicados a la venta y servicios (restaurantes, hoteles, comercio, etc.) y a los puestos que demandan poca cualificación. 


\section{Cuadro 3: Desempleados por edad y nivel de formación}

\begin{tabular}{|c|c|c|c|c|}
\hline & 2008 & 2010 & 2012 & 2014 \\
\hline 15-29, miles de parados & 1012,1 & 1566,6 & 1770,3 & 1651,6 \\
\hline Por debajo de educación & & & & 1088,2 \\
\hline Educación secundaria & & & & 204,5 \\
\hline Superior & & & & 358,9 \\
\hline Tasa de paro & $18,20 \%$ & $31,70 \%$ & $40,30 \%$ & $41,00 \%$ \\
\hline 30-54, miles de parados & 1402,9 & 2707 & 3531,1 & 3611,5 \\
\hline Por debajo de educación & & & & 2395,6 \\
\hline Educación secundaria & & & & 357 \\
\hline Superior & & & & 858,8 \\
\hline Tasa de paro & $9,40 \%$ & $17,20 \%$ & $21,90 \%$ & $22,70 \%$ \\
\hline 55 o más, miles de parados & 177,1 & 362,7 & 503,2 & 586,5 \\
\hline Por debajo de educación & & & & 532,1 \\
\hline Educación secundaria & & & & 33,4 \\
\hline Superior & & & & 69,5 \\
\hline Tasa de paro & $6,90 \%$ & $13,40 \%$ & $17,00 \%$ & $19,20 \%$ \\
\hline
\end{tabular}

Fuente: INE

Cuadro 4: Desempleados por sector y duración del desempleo

\begin{tabular}{|c|c|c|c|c|}
\hline & 2008 & 2010 & 2012 & 2014 \\
\hline Agricultura & 132,3 & 215,5 & 277,7 & 258,80 \\
\hline Hasta 3 meses & 56,7 & 83.075 & 108,05 & 101,1 \\
\hline 3 - 12 meses & 75,6 & 132,4 & 169,6 & 157,7 \\
\hline Tasa de paro & $13,77 \%$ & $21,52 \%$ & $27,20 \%$ & $25,50 \%$ \\
\hline Manufacturas & 213,1 & 266,1 & 315 & 243,10 \\
\hline Hasta 3 meses & 90,4 & 75.325 & 93,35 & 70,4 \\
\hline $3-12$ meses & 122,7 & 190,8 & 221,7 & 172,7 \\
\hline Tasa de paro & $6,18 \%$ & $9,12 \%$ & $11,26 \%$ & $9,43 \%$ \\
\hline Construcción & 422,1 & 501,9 & 429,4 & 295,20 \\
\hline Hasta 3 meses & 186,3 & 143 & 115,7 & 86,5 \\
\hline 3 - 12 meses & 235,8 & 358,9 & 313,7 & 208,7 \\
\hline Tasa de paro & $14,65 \%$ & $23,31 \%$ & $26,99 \%$ & $23,06 \%$ \\
\hline Servicios & 958,7 & 1446,8 & 1720,5 & 1574,10 \\
\hline Hasta 3 meses & 372,4 & 447,7 & 524,8 & 494,8 \\
\hline $3-12$ meses & 586,3 & 999,1 & 1195,7 & 1079,3 \\
\hline Tasa de paro & $6,43 \%$ & $9,59 \%$ & $11,50 \%$ & $10,73 \%$ \\
\hline Más de 1 año, número de & 635,4 & 1853,7 & 2572 & 2930,70 \\
\hline Tasa de paro & $2,75 \%$ & $7,93 \%$ & $10,97 \%$ & $12,73 \%$ \\
\hline Más de 2 años, número de & 256,9 & 787,7 & 1487,2 & 2324,30 \\
\hline Never in job, número de & 230,4 & 352,3 & 490 & 547,70 \\
\hline Tasa de paro & $1,00 \%$ & $1,51 \%$ & $2,09 \%$ & $2,38 \%$ \\
\hline
\end{tabular}

Fuente: INE.

Nota: La clasificación de desempleados por sectores se establece en función del sector en el que estaban ocupados cuando fueron despedidos. 


\section{Cuadro 5: Desempleados según ocupación anterior}

\begin{tabular}{lr}
\hline Total desempleados, 2014 & 5301,85 \\
\hline Directores y gerentes & $0,50 \%$ \\
Técnicos y profesionales científicos & $2,91 \%$ \\
Técnicos y profesionales de apoyo & $2,94 \%$ \\
Contables, administrativos, oficinas & $2,99 \%$ \\
Trabajadores de servicios, restauración y ventas & $11,31 \%$ \\
Trabajadores cualificados sector primario & $0,72 \%$ \\
Artesanos y trabajadores cualificados manuf. y constr. & $6,97 \%$ \\
Operadores de instalac. y maquinaria, montadores & $2,90 \%$ \\
Ocupaciones elementales & $13,47 \%$ \\
Defensa & $0,02 \%$ \\
Desempleados más de 1 año & $55,28 \%$ \\
\hline
\end{tabular}

Fuente: INE

Nota: "Total de desempleados" incluye solamente a los que han trabajado en los 12 últimos meses.

\section{El aumento del desempleo y su composición sectorial}

Una de las cuestiones que vimos en el apartado anterior es que, sobre todo en la primera fase de la crisis, España había sufrido una destrucción de empleo más elevada que la media cuando se pone en proporción a la caída del PIB. Esto plantea la cuestión de si esta mayor elasticidad empleo/PIB obedece a problemas de regulación del mercado de trabajo, lo que justificaría alguna reforma laboral para evitarlo.

El gobierno parece pensar que esto es así, porque en el preámbulo del Real Decreto de la reforma de 2012 se señala que España ha destruido más empleo, y más rápidamente, que otras economías europeas, y se considera que esto evidencia que los problemas del mercado de trabajo no son coyunturales, sino estructurales, por lo que requieren una reforma laboral de envergadura.

Sin embargo, la literatura que analiza mayor destrucción de empleo asociada a la misma caída del PIB también podría deberse, de acuerdo con la segunda de las hipótesis que enunciábamos más arriba, al peso tan elevado de la construcción en España y al fuerte impacto de la crisis sobre ese sector, o también a la extensión de los contratos fácilmente extinguibles. 
Por ejemplo, el Fondo Monetario Internacional (2010) estudia esta cuestión en el marco de la "ley de Okun" ${ }^{2}$ y encuentra en primer lugar que, aunque la relación entre crecimiento del PIB y variación de la tasa de paro -la pendiente de esta ecuación- varía mucho entre países, se observa una tendencia generalizada a su crecimiento. Y atribuye esta tendencia, en primer lugar, a las reformas de los mercados de trabajo, pero precisamente a aquellas que han aumentado un cierto tipo de "flexibilidad": la facilidad de despido y la extensión de los contratos temporales. Cuando se produce una caída de la actividad, estas dos características del mercado de trabajo se traducen en una reducción más rápida del empleo. En este sentido, la rápida pérdida de empleos con contrato temporal que se produjo en España al iniciarse la crisis sería un indicador no de un mercado de trabajo demasiado "rígido", sino al contrario, la constatación de un empleo demasiado volátil. Y los datos que hemos expuesto más arriba mostrarían que la reforma laboral no ha mitigado el problema.

En segundo lugar, también concluye que en las recesiones la pérdida de empleo suele ser mayor que lo que indica el coeficiente de la ley de Okun en aquellos casos en los que se produce una crisis financiera o una caída de los precios de la vivienda, y pone a España y Estados Unidos como ejemplo en la recesión de 2008-2009.

Por el contrario, aquellos países en los que se llevan a cabo programas de reducción (temporal) del tiempo de trabajo, como Alemania, la pérdida de puestos de trabajo (aunque no de horas de trabajo) es menor. Este tipo de políticas pueden aplicarse en aquellos países donde las instituciones laborales, la negociación colectiva y la implicación pública favorecen su rápida implantación. Pero, en nuestra opinión, también se requieren otros factores de naturaleza estructural que las hacen razonables. Por ejemplo, Alemania experimentó un shock que tenía su origen en la caída de la demanda externa, y no el colapso de un sector específico, cuya recuperación es más incierta. Además, las características de sus empresas exportadoras y la cualificación de la mano de obra hace aconsejable para las empresas "acumular" trabajadores incluso en periodos en los que el nivel de actividad es inferior al normal, por los elevados costes de reclutamiento y formación. En cambio, este incentivo es mucho menos para las empresas que se encuentran en sectores en los que se ha producido una sobredimensión

\footnotetext{
2 Recordamos que esta "ley" expresa una relación inversa entre el crecimiento del PIB y la tasa de desempleo. Sirve para determinar en cada economía cuánto debe crecer el PIB como mínimo para que el paro se reduzca, y cuánto se reduce o incrementa por cada punto en el que el PIB se sitúa por encima o por debajo de ese umbral.
} 
de la oferta durante algún tiempo (excesivo peso de la construcción en España, por ejemplo), en los que la cualificación del empleo es baja y son frecuentes los contratos temporales y una elevada rotación laboral, o cuando hay una gran incertidumbre sobre el tiempo que tardará en recuperarse el nivel de actividad previo a la crisis.

El Banco Central Europeo (2012) y Fernández e Izquierdo (2013) aplican un análisis similar al caso de los mercados de trabajo en la UE, y concluyen también que la naturaleza de los shocks que afectan a cada economía es fundamental para explicar el comportamiento del empleo. Por ejemplo, la explosión de una burbuja de la construcción suele tener un efecto más prolongado sobre la actividad, y que esto implica ajustes laborales más intensos. Junto a ello, destacan otros factores relevantes como el elevado nivel de endeudamiento previo o la importancia de los contratos temporales.

A partir de estas ideas revisamos a continuación la pérdida de empleo en España desde un punto de vista sectorial, y podemos ver que la caída sufrida por ciertos sectores económicos especialmente importantes en términos de empleo confirma la existencia de factores estructurales que explican su evolución más allá de la legislación laboral.

Dos sectores (manufacturas y construcción) representan el 80\% del total de puestos de trabajo directos perdidos (cuadro 6). En concreto, el empleo en la construcción se ha reducido hasta 2014 en un 64\% respecto al nivel que tenía en 2007, y la reducción también ha sido significativa en el sector de las manufacturas (con una pérdida aproximada del $30 \%$ de sus puestos de trabajo). En cuanto al empleo directamente relacionado con los servicios públicos, si bien continuó aumentando hasta finales de 2011, a partir de entonces se ha observado una considerable reducción como consecuencia de las políticas de ajuste presupuestario. Tal como se puede observar en el cuadro 7, la media del empleo anual en los sectores de administración pública, defensa, educación y servicios sanitarios sufrió una reducción de 256.000 empleos entre 2011 y 2014.

El impacto del declive de la construcción en el incremento del desempleo en España es incluso más importante si tenemos en cuenta los "multiplicadores de empleo" de cada uno de los sectores económicos, en los que se recoge el efecto que tiene el incremento de la producción de un sector económico en el empleo del resto de sectores. 


\section{Cuadro 6: Empleo por sector de actividad}

\begin{tabular}{|c|c|c|c|c|c|}
\hline \multicolumn{2}{|l|}{ Actividades NACE 2} & \multicolumn{4}{|c|}{ Miles } \\
\hline & & 2007 & 2009 & 2011 & 2014 \\
\hline $\begin{array}{l}\text { Agricultura, ganadería, } \\
\text { silvicultura y pesca }\end{array}$ & $\mathrm{A}$ & 866,2 & 788,1 & 755,3 & 738,2 \\
\hline Industrias extractivas & $\mathrm{B}$ & 59,3 & 44,4 & 42,0 & 32,5 \\
\hline Manufacturas & $\mathrm{C}$ & 2995,5 & 2549,4 & 2349,7 & 2123,8 \\
\hline $\begin{array}{l}\text { Suministros de energía eléctrica, } \\
\text { gas y agua }\end{array}$ & $\mathrm{D}-\mathrm{E}$ & 201,7 & 213,8 & 213,0 & 204,0 \\
\hline Construcción + real estate & $\mathrm{F}$ & 2759,2 & 1889,8 & 1403,9 & 981,2 \\
\hline $\begin{array}{l}\text { Comercio al por mayor y al por } \\
\text { menor }\end{array}$ & $\mathrm{G}$ & 3116,0 & 2990,5 & 2962,5 & 2858,2 \\
\hline Transporte & $\mathrm{H}$ & 964,6 & 923,8 & 899,3 & 852,7 \\
\hline $\begin{array}{l}\text { Actividades de alojamiento y } \\
\text { hostelería }\end{array}$ & $\mathrm{I}$ & 1456,6 & 1423,3 & 1401,1 & 1405,0 \\
\hline Información y comunicaciones & $\mathrm{J}$ & 567,2 & 533,3 & 533,3 & 508,5 \\
\hline $\begin{array}{l}\text { Actividades financieras y de } \\
\text { seguros }\end{array}$ & $\mathrm{K}$ & 508,0 & 487,5 & 464,6 & 453,7 \\
\hline Actividades inmobiliarias & $\mathrm{L}$ & 108,5 & 91,9 & 96,3 & 100,5 \\
\hline $\begin{array}{l}\text { Actividades profesionales, } \\
\text { científicas y técnicas; } \\
\text { administrativas y servicios } \\
\text { auxiliares }\end{array}$ & M-N & 1814,8 & 1793,4 & 1767,7 & 1733,0 \\
\hline $\begin{array}{l}\text { Administración pública y } \\
\text { defensa. Seguridad social }\end{array}$ & $\mathrm{O}$ & 1257,6 & 1387,8 & 1452,8 & 1302,0 \\
\hline Educación & $\mathrm{P}$ & 1147,8 & 1180,1 & 1206,1 & 1142,8 \\
\hline $\begin{array}{l}\text { Actividades sanitarias y de } \\
\text { servicios sociales }\end{array}$ & $\mathrm{Q}$ & 1243,3 & 1352,6 & 1462,5 & 1420,6 \\
\hline $\begin{array}{l}\text { Actividades artísticas, } \\
\text { recreativas y de entretenimiento; } \\
\text { otros }\end{array}$ & $\mathrm{R}-\mathrm{U}$ & 1513,4 & 1457,3 & 1411,6 & 1412,5 \\
\hline Total & $\mathrm{A}-\mathrm{U}$ & 20579,6 & 19106,9 & 18421,4 & 17269,2 \\
\hline $\begin{array}{l}\text { Construcción + actividades } \\
\text { inmobiliarias }\end{array}$ & $\mathrm{F}+\mathrm{L}$ & 2867,7 & 1981,7 & 1500,2 & 1081,7 \\
\hline $\begin{array}{l}\text { Administración pública, defensa, } \\
\text { educación, sanidad y servicios } \\
\text { sociales }\end{array}$ & $\mathrm{O}-\mathrm{Q}$ & 3648,6 & 3920,5 & 4121,4 & 3865,4 \\
\hline
\end{tabular}




\section{Cuadro 6 (continuación)}

\begin{tabular}{|c|c|c|c|c|c|c|c|}
\hline \multicolumn{2}{|l|}{ Actividades NACE 2} & \multicolumn{3}{|c|}{ Cambio entre 2007 y 2014} & \multicolumn{3}{|c|}{ Cambio entre 2011 y 2014} \\
\hline & & Miles & $\%$ & $\begin{array}{c}\% \text { del } \\
\text { total }\end{array}$ & Miles & $\%$ & $\begin{array}{c}\% \text { del } \\
\text { total }\end{array}$ \\
\hline $\begin{array}{l}\text { Agricultura, ganadería, silvicultura y } \\
\text { pesca }\end{array}$ & $\mathrm{A}$ & $-128,1$ & $-14,8 \%$ & $3,9 \%$ & $-17,1$ & $-2,3 \%$ & $1,5 \%$ \\
\hline Industrias extractivas & $\mathrm{B}$ & $-26,9$ & $-45,3 \%$ & $0,8 \%$ & $-9,6$ & $-22,7 \%$ & $0,8 \%$ \\
\hline Manufacturas & $\mathrm{C}$ & $-871,7$ & $-29,1 \%$ & $26,3 \%$ & $-225,9$ & $-9,6 \%$ & $19,6 \%$ \\
\hline $\begin{array}{l}\text { Suministros de energía eléctrica, gas y } \\
\text { agua }\end{array}$ & D-E & 2,3 & $1,2 \%$ & $-0,1 \%$ & $-9,0$ & $-4,2 \%$ & $0,8 \%$ \\
\hline Construcción + real estate & $\mathrm{F}$ & $-1778,0$ & $-64,4 \%$ & $53,7 \%$ & $-422,7$ & $-30,1 \%$ & $36,7 \%$ \\
\hline Comercio al por mayor y al por menor & $\mathrm{G}$ & $-257,8$ & $-8,3 \%$ & $7,8 \%$ & $-104,3$ & $-3,5 \%$ & $9,1 \%$ \\
\hline Transporte & $\mathrm{H}$ & $-111,9$ & $-11,6 \%$ & $3,4 \%$ & $-46,6$ & $-5,2 \%$ & $4,0 \%$ \\
\hline Actividades de alojamiento y hostelería & I & $-51,6$ & $-3,5 \%$ & $1,6 \%$ & 3,9 & $0,3 \%$ & $-0,3 \%$ \\
\hline Información y comunicaciones & $\mathrm{J}$ & $-58,7$ & $-10,3 \%$ & $1,8 \%$ & $-24,8$ & $-4,6 \%$ & $2,2 \%$ \\
\hline Actividades financieras y de seguros & $\mathrm{K}$ & $-54,3$ & $-10,7 \%$ & $1,6 \%$ & $-10,9$ & $-2,3 \%$ & $0,9 \%$ \\
\hline Actividades inmobiliarias & $\mathrm{L}$ & $-8,0$ & $-7,3 \%$ & $0,2 \%$ & 4,3 & $4,4 \%$ & $-0,4 \%$ \\
\hline $\begin{array}{l}\text { Actividades profesionales, científicas y } \\
\text { técnicas; administrativas y servicios } \\
\text { auxiliares }\end{array}$ & $\mathrm{M}-\mathrm{N}$ & $-81,8$ & $-4,5 \%$ & $2,5 \%$ & $-34,7$ & $-2,0 \%$ & $3,0 \%$ \\
\hline $\begin{array}{l}\text { Administración pública y defensa. } \\
\text { Seguridad social }\end{array}$ & $\mathrm{O}$ & 44,5 & $3,5 \%$ & $-1,3 \%$ & $-150,7$ & $-10,4 \%$ & $13,1 \%$ \\
\hline Educación & $\mathrm{P}$ & $-5,0$ & $-0,4 \%$ & $0,2 \%$ & $-63,4$ & $-5,3 \%$ & $5,5 \%$ \\
\hline $\begin{array}{l}\text { Actividades sanitarias y de servicios } \\
\text { sociales }\end{array}$ & $\mathrm{Q}$ & 177,3 & $14,3 \%$ & $-5,4 \%$ & $-41,9$ & $-2,9 \%$ & $3,6 \%$ \\
\hline $\begin{array}{l}\text { Actividades artísticas, recreativas y de } \\
\text { entretenimiento; otros }\end{array}$ & $\mathrm{R}-\mathrm{U}$ & $-100,9$ & $-6,7 \%$ & $3,0 \%$ & 1,0 & $0,1 \%$ & $-0,1 \%$ \\
\hline Total & $\mathrm{A}-\mathrm{U}$ & $-3310,4$ & $-16,1 \%$ & $100,0 \%$ & $-1152,2$ & $-6,3 \%$ & $100,0 \%$ \\
\hline Construcción + actividades inmobiliarias & $\mathrm{F}+\mathrm{L}$ & $-1785,9$ & $-62,3 \%$ & $53,9 \%$ & $-418,4$ & $-27,9 \%$ & $36,3 \%$ \\
\hline $\begin{array}{l}\text { Administración pública, defensa, } \\
\text { educación, sanidad y servicios sociales }\end{array}$ & $\mathrm{O}-\mathrm{Q}$ & 216,8 & $5,9 \%$ & $-6,5 \%$ & $-256,0$ & $-6,2 \%$ & $22,2 \%$ \\
\hline
\end{tabular}

Fuente: INE

\section{Cuadro 7: Empleo público y privado (miles de puestos de trabajo)}

\begin{tabular}{lrrrr}
\hline & \multicolumn{1}{c}{2008} & \multicolumn{1}{c}{2010} & \multicolumn{1}{c}{2012} & \multicolumn{1}{c}{2014} \\
\hline Empleo público & 3006,7 & 3209,6 & 3112,4 & 2925,7 \\
Empleo privado & 17463,0 & 15514,9 & 14520,3 & 14241,6 \\
\hline
\end{tabular}

Fuente: INE 
Para medir este efecto calculamos previamente la cantidad de empleo total que se requiere para satisfacer la demanda final de cada uno de los bienes y servicios obtenidos -también denominado "empleo verticalmente integrado" en la terminología de Pasinetti (1973)-, que llamamos $\mathbf{l}_{t}$. Utilizando la metodología tradicional input-output y los datos contenidos en la WIOD (Timmer et al. 2012), el empleo total se obtiene mediante la siguiente ecuación:

$$
\mathbf{l}_{t}=\mathbf{a}_{\mathbf{n} t}[\mathbf{I}-\mathbf{A}]^{-\mathbf{1}} \cdot\langle\mathbf{y}>
$$

donde $\mathbf{a}_{\mathbf{n}}$ es el vector que contiene los coeficientes directos de trabajo correspondientes a la obtención de un millón de euros de producción total de cada sector económico, $[\mathbf{I}-\mathbf{A}]^{\mathbf{1}}$ es la expresión tradicional de la inversa de Leontief y $\langle\mathbf{y}\rangle$ es una matriz diagonal calculada a partir del vector $\mathbf{y}$ que contiene la demanda final en términos físicos que se produce anualmente en cada sector económico, y que está formado por el consumo público y privado, las exportaciones, la formación bruta de capital fijo y la variación de existencias.

El cuadro 8 contiene los resultados del empleo para 2007, 2009 y 2011. Los datos muestran el empleo directo y el empleo total de acuerdo a la expresión anterior, con un nivel de desagregación de 16 sectores. Dado que no hay datos input-output disponibles a partir de 2011, el trabajo verticalmente integrado para 2014 ha sido estimado multiplicando los datos directos de empleo de 2014 por el multiplicador de empleo ${ }^{3}$ obtenido en 2011.

De acuerdo con los resultados obtenidos, cuatro sectores verticalmente integrados acumulan el 56\% del empleo total en 2007: Manufacturas (19\%), Construcción y servicios inmobiliarios (18\%), actividades de alojamiento y hostelería (10\%), y comercio mayorista y minorista (9\%). Además, otro $20 \%$ del empleo estaba relacionado con actividades del sector público (educación y sanidad) ${ }^{4}$ o administración pública y defensa.

El cuadro 8 muestra también los encadenamientos hacia adelante y hacia atrás que existen entre los diferentes sectores económicos. En 2007, 6,4 millones de trabajadores estuvieron ocupados en la producción de los bienes y servicios que son utilizados como inputs

\footnotetext{
${ }^{3}$ Ratio entre el trabajo verticalmente integrado que está asociado a la demanda final de un sector económico y el empleo directo en el mismo sector económico.

4 Aunque un porcentaje muy elevado de los servicios de educación y sanidad son proporcionados por el sector público, no todos estos trabajadores pertenecen a la administración
} 
por el resto de sectores económicos (columna "out" del cuadro 8). Tal como se observa en dicha columna, la construcción es uno de los sectores donde el trabajo que se requiere para satisfacer el consumo intermedio del resto de sectores (es decir, el encadenamiento hacia adelante) es menor que el trabajo inducido en el resto de los sectores para satisfacer su propia demanda (la columna "ind", donde aparece la medida de los encadenamientos hacia atrás). Estas cifras ponen de manifiesto la capacidad del sector de la construcción para aumentar el empleo en otros sectores económicos. Y esto también es cierto en otras actividades que también se han visto muy afectadas por la crisis, como la Industria manufacturera, los servicios proporcionados desde el sector público (después de 2011) y los servicios de alojamiento y restauración. En el extremo opuesto, las actividades de servicios profesionales, administrativas y servicios auxiliares, transportes e industrias extractivas, muestran una fuerte dependencia del resto de sectores económicos respecto a la adquisición de bienes intermedios.

Todo lo anterior pone de relieve una vez más la alta especialización de la economía española en las actividades de construcción y servicios inmobiliarios durante los últimos años, así como el gran impacto que el estallido de la burbuja inmobiliaria ha producido sobre el empleo total. Según el cuadro 9, la reducción en el número de puestos de trabajo que están directa o indirectamente asociados a la vivienda alcanzó 2,3 millones en términos absolutos entre 2007 y 2014, lo que supone un $69 \%$ de la reducción total en el empleo de la economía española. Este efecto es aún más evidente entre 2007 y 2009, en que la pérdida de empleos relacionados con la construcción supuso el $82 \%$ de la reducción en el empleo de la economía en su conjunto.

Esta caída en el empleo que está directa e indirectamente relacionado con la construcción no ha estado motivada por la existencia de salarios demasiado altos, de modo que la flexibilidad salarial no podría haber evitado este problema. Las causas habría que buscarlas en los desequilibrios que la economía española había acumulado durante el período de auge anterior a la recesión económica $\mathrm{y}$, de manera más específica, en un elevado endeudamiento del sector privado y en una excesiva dimensión de la construcción. 


\section{Cuadro 8: Empleo directo e indirecto en España (2007-2011-2014, miles de puestos de trabajo)}

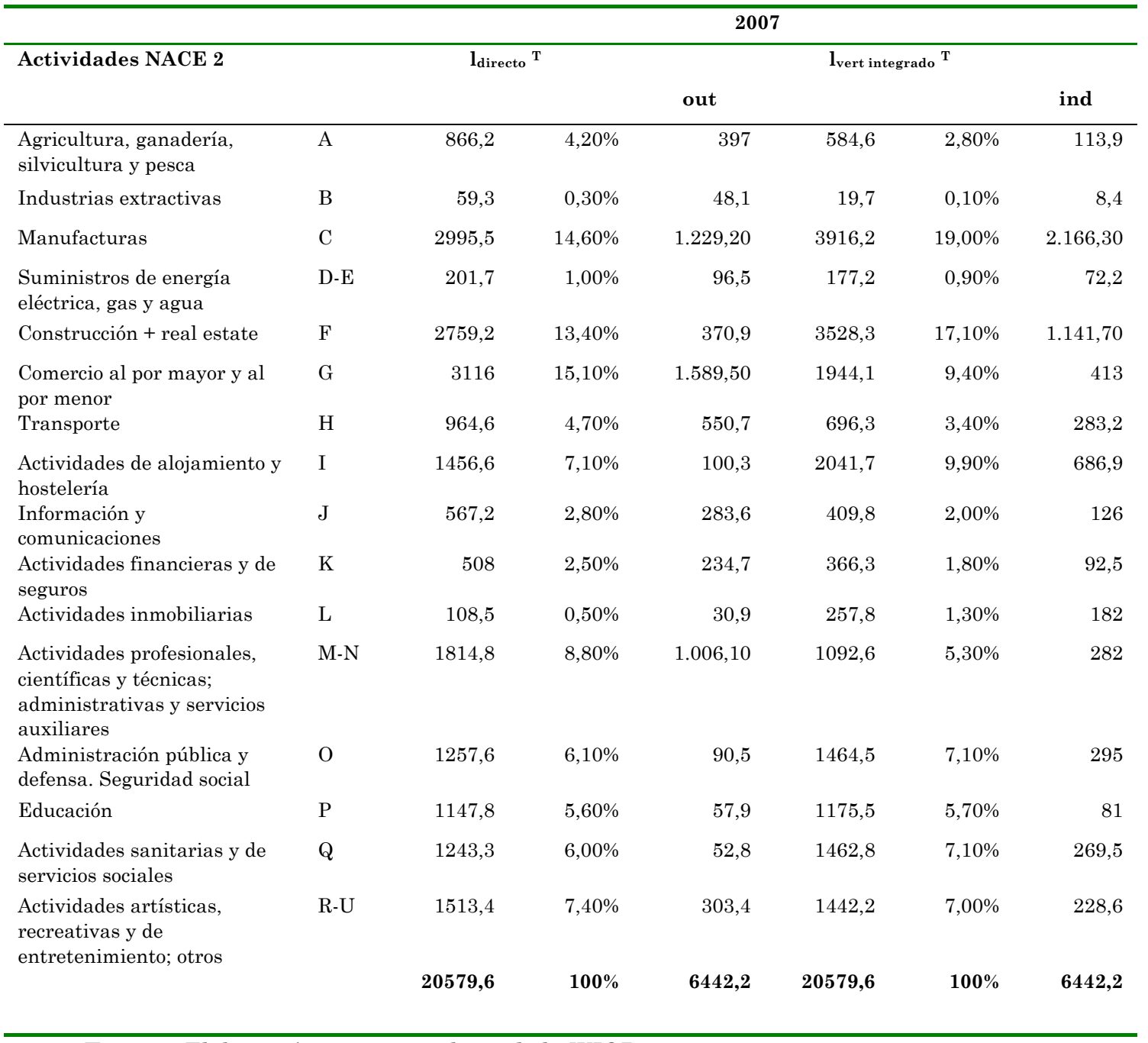

Fuente: Elaboración propia con datos de la WIOD.

Nota: ldirecto - Número de trabajadores necesarios para obtener el output total de cada sector económico, de los cuales out contiene los empleados que corresponden a la parte de la producción destinada al consumo intermedio del resto de sectores económicos. Tomando como ejemplo el sector de Agricultura, silvicultura y pesca, en 2007 se contabilizaron 866,2 (miles) empleos totales, de los cuales, 397 (miles) correspondieron a la producción de este sector que fue adquirida por el resto de sectores como bienes intermedios. 


\section{Cuadro 8 (continuación)}

\begin{tabular}{|c|c|c|c|c|c|c|c|c|c|c|c|}
\hline \multicolumn{6}{|c|}{2011} & \multicolumn{6}{|c|}{2014} \\
\hline \multicolumn{2}{|c|}{$l_{\text {directo }} T$} & \multicolumn{4}{|c|}{$l_{\text {vert integrado }}{ }^{T}$} & \multicolumn{2}{|c|}{$l_{\text {directo }} T$} & \multicolumn{4}{|c|}{$l_{\text {vert integrado }} T$} \\
\hline & & out & & & ind & & & out & & & ind \\
\hline 755,3 & $4,10 \%$ & 343,6 & 517,1 & $2,80 \%$ & 105,4 & 738,2 & $4,30 \%$ & 332,9 & 511 & $3,00 \%$ & 105,7 \\
\hline 42 & $0,20 \%$ & 30,1 & 21 & $0,10 \%$ & 9,1 & 32,5 & $0,20 \%$ & 22,9 & 16,4 & $0,10 \%$ & 6,8 \\
\hline 2349,7 & $12,80 \%$ & 818,2 & 3579,1 & $19,40 \%$ & $2.048,10$ & 2123,8 & $12,30 \%$ & 719 & 3270,9 & $18,90 \%$ & $1.866,10$ \\
\hline 213 & $1,20 \%$ & 107,3 & 190,1 & $1,00 \%$ & 84,5 & 204 & $1,20 \%$ & 101,7 & 184,1 & $1,10 \%$ & 81,9 \\
\hline 1403,9 & $7,60 \%$ & 230,7 & 1867 & $10,10 \%$ & 693,9 & 981,2 & $5,70 \%$ & 154 & 1319,3 & $7,60 \%$ & 492,1 \\
\hline 2962,5 & $16,10 \%$ & $1.498,50$ & 1846,5 & $10,00 \%$ & 382,3 & 2858,2 & $16,60 \%$ & $1.430,50$ & 1801,3 & $10,40 \%$ & 373,6 \\
\hline 899,3 & $4,90 \%$ & 492,9 & 696,1 & $3,80 \%$ & 289,7 & 852,7 & $4,90 \%$ & 459,6 & 667,3 & $3,90 \%$ & 274,2 \\
\hline 1401,1 & $7,60 \%$ & 99,6 & 1925 & $10,40 \%$ & 623,6 & 1405 & $8,10 \%$ & 75,9 & 1951,8 & $11,30 \%$ & 622,7 \\
\hline 533,3 & $2,90 \%$ & 272,9 & 371,2 & $2,00 \%$ & 110,8 & 508,5 & $2,90 \%$ & 262,8 & 357,9 & $2,10 \%$ & 112,2 \\
\hline 464,6 & $2,50 \%$ & 200 & 369,2 & $2,00 \%$ & 104,6 & 453,7 & $2,60 \%$ & 193,2 & 364,6 & $2,10 \%$ & 104,1 \\
\hline 96,3 & $0,50 \%$ & 28,6 & 236,7 & $1,30 \%$ & 169 & 100,5 & $0,60 \%$ & 27,1 & 249,7 & $1,40 \%$ & 176,3 \\
\hline 1767,7 & $9,60 \%$ & $1.039,70$ & 943,6 & $5,10 \%$ & 215,4 & 1733 & $10,00 \%$ & $1.012,40$ & 935,3 & $5,40 \%$ & 214,7 \\
\hline 1452,8 & $7,90 \%$ & 105 & 1638,4 & $8,90 \%$ & 290,7 & 1302 & $7,50 \%$ & 79,6 & 1484,6 & $8,60 \%$ & 262,2 \\
\hline 1206,1 & $6,50 \%$ & 59 & 1225,6 & $6,70 \%$ & 78,4 & 1142,8 & $6,60 \%$ & 44,2 & 1174,2 & $6,80 \%$ & 75,6 \\
\hline 1462,5 & $7,90 \%$ & 59,4 & 1668 & $9,10 \%$ & 264,9 & 1420,6 & $8,20 \%$ & 41,2 & 1638,2 & $9,50 \%$ & 258,8 \\
\hline 1411,6 & $7,70 \%$ & 285,4 & 1326,9 & $7,20 \%$ & 200,6 & 1412,5 & $8,20 \%$ & 269,7 & 1342,5 & $7,80 \%$ & 199,7 \\
\hline 18421,6 & $100 \%$ & 5671,0 & 18421,6 & $100 \%$ & 5671,0 & 17269,2 & $100 \%$ & 5226,6 & 17269,2 & $100 \%$ & 5226,6 \\
\hline
\end{tabular}

Fuente: Elaboración propia con datos de la WIOD.

Nota: lvert integrado - Número de empleos vinculados a la producción de la demanda final de cada sector económico, de los cuales ind contiene el número de empleos inducidos en el resto de sectores económicos. Por ejemplo: el número de puestos de trabajo que se necesitaron en 2007 para satisfacer la demanda final del sector de Agricultura, silvicultura y pesca fue 584,6 (miles), de los cuales 113,9 (miles) correspondieron a los puestos de trabajo del resto de sectores económicos ocupados en la producción utilizada como bienes intermedios en el sector de Agricultura, ganadería, silvicultura y pesca. 


\section{Cuadro 9: Variación en el empleo verticalmente integrado}

\begin{tabular}{|c|c|c|c|c|c|c|c|}
\hline \multirow[t]{2}{*}{ Actividades NACE 2} & & \multicolumn{3}{|c|}{$\begin{array}{c}\text { Cambio entre } 2007 \mathrm{y} \\
2011\end{array}$} & \multicolumn{3}{|c|}{$\begin{array}{c}\text { Cambio entre } 2011 \mathrm{y} \\
2014\end{array}$} \\
\hline & & Miles & $\%$ & $\begin{array}{c}\% \text { del } \\
\text { total }\end{array}$ & Miles & $\%$ & $\begin{array}{c}\% \text { del } \\
\text { total }\end{array}$ \\
\hline $\begin{array}{l}\text { Agricultura, ganadería, } \\
\text { silvicultura y pesca }\end{array}$ & A & $-67,5$ & $-11,5$ & 3,1 & $-6,1$ & $-1,2$ & 0,5 \\
\hline Industrias extractivas & B & 1,3 & 6,8 & $-0,1$ & $-4,6$ & $-21,8$ & 0,4 \\
\hline Manufacturas & $\mathrm{C}$ & $-337,1$ & $-8,6$ & 15,6 & $-308,2$ & $-8,6$ & 26,7 \\
\hline $\begin{array}{l}\text { Suministros de energía } \\
\text { eléctrica, gas y agua }\end{array}$ & D-E & 13,0 & 7,3 & $-0,6$ & $-6,0$ & $-3,2$ & 0,5 \\
\hline $\begin{array}{l}\text { Construcción + real } \\
\text { estate }\end{array}$ & $\mathrm{F}$ & $-1661,4$ & $-47,1$ & 77,0 & $-547,6$ & $-29,3$ & 47,5 \\
\hline $\begin{array}{l}\text { Comercio al por mayor y } \\
\text { al por menor }\end{array}$ & $\mathrm{G}$ & $-97,6$ & $-5,0$ & 4,5 & $-45,2$ & $-2,4$ & 3,9 \\
\hline Transporte & $\mathrm{H}$ & $-0,3$ & 0,0 & 0,0 & $-28,7$ & $-4,1$ & 2,5 \\
\hline $\begin{array}{l}\text { Actividades de } \\
\text { alojamiento y hostelería }\end{array}$ & I & $-116,7$ & $-5,7$ & 5,4 & 26,8 & 1,4 & $-2,3$ \\
\hline $\begin{array}{l}\text { Información y } \\
\text { comunicaciones }\end{array}$ & $\mathrm{J}$ & $-38,6$ & $-9,4$ & 1,8 & $-13,3$ & $-3,6$ & 1,2 \\
\hline $\begin{array}{l}\text { Actividades financieras y } \\
\text { de seguros }\end{array}$ & $\mathrm{K}$ & 2,9 & 0,8 & $-0,1$ & $-4,7$ & $-1,3$ & 0,4 \\
\hline Actividades inmobiliarias & $\mathrm{L}$ & $-21,1$ & $-8,2$ & 1,0 & 13,1 & 5,5 & $-1,1$ \\
\hline $\begin{array}{l}\text { Actividades profesionales, } \\
\text { científicas y técnicas; } \\
\text { administrativas y } \\
\text { servicios auxiliares }\end{array}$ & $\begin{array}{l}\mathrm{M}- \\
\mathrm{N}\end{array}$ & $-149,1$ & $-13,6$ & 6,9 & $-8,2$ & $-0,9$ & 0,7 \\
\hline $\begin{array}{l}\text { Administración pública y } \\
\text { defensa. Seguridad social }\end{array}$ & $\mathrm{O}$ & 173,9 & 11,9 & $-8,1$ & $-153,8$ & $-9,4$ & 13,3 \\
\hline Educación & $\mathrm{P}$ & 50,1 & $4,3 \%$ & $-2,3 \%$ & $-51,4$ & -4 & 4,5 \\
\hline $\begin{array}{l}\text { Actividades sanitarias y } \\
\text { de servicios sociales }\end{array}$ & Q & 205,2 & 14,0 & $-9,5$ & $-29,8$ & $-1,8$ & 2,6 \\
\hline $\begin{array}{l}\text { Actividades artísticas, } \\
\text { recreativas y de } \\
\text { entretenimiento; otros }\end{array}$ & $\mathrm{R}-\mathrm{U}$ & $-115,3$ & $-8,0$ & 5,3 & 15,6 & 1,2 & $-1,4$ \\
\hline Total & $\mathrm{A}-\mathrm{U}$ & $-2158,2$ & $-10,5$ & 100,0 & $-1152,2$ & $-6,3$ & 100,0 \\
\hline $\begin{array}{l}\text { Construcción }+ \\
\text { actividades inmobiliarias }\end{array}$ & $\begin{array}{l}\mathrm{F}+ \\
\mathrm{L}\end{array}$ & $-1682,5$ & $-44,4$ & 78,0 & $-534,6$ & $-25,4$ & 46,4 \\
\hline $\begin{array}{l}\text { Administración pública, } \\
\text { defensa, educación, } \\
\text { sanidad y servicios } \\
\text { sociales }\end{array}$ & $\mathrm{O}-\mathrm{Q}$ & 429,2 & 10,5 & $-19,9$ & $-235,0$ & $-5,2$ & 20,4 \\
\hline
\end{tabular}

Fuente: Elaboración propia con datos de la WIOD.

En 1997, el endeudamiento de los hogares, sobre todo el destinado a financiar la compra de una vivienda, comenzó a crecer. Tal como indican Dejuán y Febrero (2011), esto se debió a una serie de 
factores demográficos (los "baby boomers" empezaron a alcanzar la edad de 30 años y la tasa de propiedad de las viviendas en España es alta) y a otras causas relacionadas con la creación de la eurozona (caída en la tasa de interés y un aumento de los movimientos del capital financiero). Desde el lado de la oferta, se produjo un enorme aumento del número de viviendas construidas, aunque al mismo tiempo los precios subían significativamente, dando lugar a la aparición de una importante actividad especulativa.

El sector de la construcción aumentó dos veces más que el PIB entre 1997 y 2007 lo cual supone un ritmo de crecimiento muy difícil de mantener. En esta década, se crearon 6,25 millones de viviendas de nueva construcción y uno de cada cinco nuevos puestos de trabajo pertenecía a este sector. En 2007, el peso de la construcción era mucho más grande que en la Unión Europea, y su crecimiento había sido compensado por una caída similar en el sector manufacturero. Sin embargo, este patrón de crecimiento se había agotado en 2007 por varias razones (Uxó, Paúl y Febrero, 2011).

En primer lugar, porque la deuda de los hogares había alcanzado niveles muy altos y los precios de la vivienda se habían triplicado en diez años. Si bien el endeudamiento supone inicialmente un mecanismo de impulso para la demanda agregada, posteriormente se puede convertir en un problema si la relación deuda-renta disponible se eleva demasiado y la pérdida de confianza de los prestamistas limita la refinanciación del servicio de la deuda de los prestatarios. Los hogares estaban excesivamente endeudados en 2007, alcanzando valores del 85\% del PIB, cuando 10 años antes su deuda era el 35\% del PIB. En este sentido, los problemas para refinanciar su deuda se agravaron con la significativa reducción del crédito que se produjo cuando el BCE decidió implantar políticas restrictivas y los tipos de interés subieron desde el 2\% en Noviembre de 2005 hasta el 4,25\% en Julio de2008.

En segundo lugar, el mercado de la vivienda comenzó a dar muestras de un nivel de saturación importante: casi 7 millones de viviendas se habían construido en la última década y, en 2007, se iniciaron 700.000 unidades más, cuando todavía quedaban 500.000 viviendas sin vender. En 2009, el stock de viviendas en venta ascendía a 688.000 unidades, un $2,7 \%$ del stock total de las viviendas existentes. Aun así, en 2007 el precio del metro cuadrado de una vivienda nueva era un $23 \%$ más elevado que en 2005 y un $200 \%$ más que en 1997 , pero la demanda comenzó a disminuir por la dificultad de acceso al crédito y la reducción de ingresos en los hogares.

En estas condiciones, la recuperación económica no podía sustentarse en la construcción. Por tanto, el verdadero problema fue 
que una vez que la construcción frenó su impulso, ningún otro sector productivo asumió la función de locomotora de la economía, debido a la falta de demanda agregada. Esto dio lugar, a su vez, a un aumento del desempleo con el que se acrecentó enormemente el problema del endeudamiento de los hogares. Como veremos en la siguiente sección, la contención salarial y la austeridad fiscal agravaron aún más la situación. Los únicos sectores en los que el empleo registró un aumento significativo entre 2007 y 2011 fueron los de la administración pública, educación y servicios de salud, cuya participación conjunta en el empleo total pasó del $20 \%$ al $25 \%$. Sin embargo, esta tendencia positiva fue interrumpida en 2011 debido a la adopción de las medidas de austeridad fiscal.

El cuadro 9 muestra también un descenso generalizado en el empleo relacionado con la industria manufacturera, donde sólo el sector del suministro de electricidad, gas y agua mostró una leve tendencia positiva durante 2007-2009. Una tendencia similar se observa en los sectores del alojamiento y restauración, donde se incluyen las actividades turísticas, el otro gran sector donde la economía española presenta una alta especialización. Sin embargo, en este caso la reducción en el empleo ha sido mucho menos significativa $(5,3 \%$ en $2007-2011$ y $0,1 \%$ en $2011-2014$ ).

\section{Devaluación interna, austeridad fiscal y empleo}

En la sección anterior hemos abordado en qué medida la mayor caída del empleo (en relación al PIB) que se observa en España en 20082009 se podía achacar a la rigidez de su mercado de trabajo y requería, en consecuencia, una reforma laboral. Hemos visto que, en realidad, la importancia de la crisis del sector de la construcción es un factor fundamental para entender esa circunstancia, tal y como señala la propia literatura que hemos referenciado.

A partir de 2011, el paro también aumenta más en España que en la media de la UE, pero nos encontramos con una situación diferente por dos razones. En primer lugar, se ponen en marcha tres reformas laborales y los salarios inician una clara senda descendente, lo que no había ocurrido en los años anteriores. En segundo lugar, el peor comportamiento relativo del empleo en España está asociado fundamentalmente con un peor comportamiento del PIB.

En esta sección revisamos cuál es la influencia que ha tenido la lo política macroeconómica en estos resultados, y en particular a la combinación de austeridad fiscal y devaluación interna, para la cual la 
reforma laboral de 2012 es completamente funcional. Es decir, una de las consecuencias de la reforma laboral (de hecho, uno de sus objetivos) ha sido modificar las condiciones de la negociación colectiva y favorecer una bajada salarial. Por tanto, queremos saber cuál ha sido el efecto que esto ha tenido sobre el empleo, y adelantamos que nuestra respuesta es que ha sido negativo. La suma de los recortes fiscales con una reforma laboral que ha propiciado una importante devaluación salarial ha tenido un importante impacto restrictivo sobre la demanda interna, pero no se ha traducido en un incremento suficiente de la demanda externa para compensarlo. El resultado ha sido un aumento del desempleo.

\subsection{La politica macroeconómica aplicada}

La política económica que el gobierno español puso en marcha en los primeros años de la Gran Recesión (2008-2009) estaba dirigida a la recuperación de la demanda interna, y se concretó en un programa de expansión fiscal alineado con las propuestas del G-20, el Plan Europeo de Recuperación Económica y el FMI. De hecho, las medidas de estímulo fiscal adoptadas en España (equivalentes al 2,3\% del PIB en 2009) estaban entre las más expansivas del mundo, porque España contaba entonces con un amplio margen fiscal (superávit del $2 \%$ en 2007 y deuda por debajo del 40\%). Como consecuencia de estas medidas, pero sobre todo por la propia intensidad de la crisis económica y sus efectos sobre los ingresos y gastos públicos, se produjo un importante aumento del déficit $(-11,1 \%$ en 2009$)$ y de la deuda pública $(53,1 \%$ en 2009, lo que supuso un aumento de 17 puntos en dos años).

A comienzos de 2010, sin embargo, esta orientación de la política macroeconómica se modifica sustancialmente, no sólo en España, sino en el conjunto de la UE. En la política presupuestaria, el cambio consiste básicamente en el abandono de estos planes de estímulo fiscal discrecional, para centrar todos los esfuerzos en el cumplimiento de las normas de disciplina presupuestaria, que son incluso reforzadas y endurecidas. En abril de 2009 se había abierto un Procedimiento de Déficit Excesivo contra España, y a partir de mayo de 2010 se aprueban sucesivos programas de ajuste con el fin de situar el déficit presupuestario por debajo del 3\%, primero en $2013 \mathrm{y}$, tras dos aplazamientos, en 2014 y 2016, que es el objetivo actual. Este cambio de orientación supone que desde ese momento la política fiscal haya pasado a ser fuertemente restrictiva y procíclica.

Inicialmente, las autoridades argumentaron que la consolidación sería expansiva, gracias a sus efectos "no keynesianos" sobre la 
demanda privada, originados por un aumento de la confianza, una mejora de las expectativas y una bajada de los tipos de interés. Sin embargo, la realidad ha mostrado que esta política fiscal sí ha tenido un fuerte impacto negativo sobre el crecimiento, lo que llevó al Fondo Monetario Internacional (2012) a reconsiderar el tamaño de los multiplicadores fiscales que estaba suponiendo. Después de señalar que la actividad económica se había comportado peor de lo esperado en las economías que adoptaron medidas de consolidación fiscal, encontró una correlación negativa significativa entre el error en las previsiones de crecimiento realizadas antes de la aplicación de las políticas de consolidación fiscal y el grado en que estas políticas fiscales estaban siendo restrictivas, y concluyó que los multiplicadores implícitos que estaban utilizándose (en torno a 0,5) eran demasiado bajos: en media, los multiplicadores reales se situarían en torno a 1,5 .

El gobierno español también reconoció que los efectos de la consolidación fiscal sobre el crecimiento a corto plazo son negativos, pasando a enfatizar en cambio los efectos positivos que se esperan a más largo plazo, cuando se combinan con otras reformas estructurales (Gobierno español, 2013, pág. 5). Y para compensar esta influencia negativa sobre la demanda interna, la estrategia de política económica se complementa con la devaluación interna, favorecida por la reforma laboral.

La devaluación interna es una estrategia para ganar competitividad a través de la consecución de tasas de inflación más bajas que los competidores, lo que en el seno de una unión monetaria sustituiría a una imposible devaluación nominal (Alexiou y Nellis, 2013). Además, en el caso de la zona euro se ha impuesto la idea de que este reajuste de los tipos de cambio reales debería ser el resultado de una reducción de los salarios en los países que tenían desequilibrio externo, como España. Si tiene éxito, se argumenta, esta política no sólo permitiría corregir los desequilibrios por cuenta corriente, sino iniciar un proceso de crecimiento basado en el tirón de las exportaciones que permitiría compensar la debilidad de la demanda interna (agravada por la propia política económica). En el contexto de esta estrategia, una de las funciones de la reforma laboral de 2012 es favorecer el proceso de ajuste salarial a la baja.

De forma alternativa a este planteamiento, Febrero y Bermejo (2013) ofrecen una interpretación diferente de las causas que condujeron a la economía española a la recesión y señalan las limitaciones de estas políticas económicas. A continuación, partiendo de ese mismo planteamiento, vamos a analizar el papel desempeñado por la devaluación salarial en la evolución de la competitividad y en el 
crecimiento de la demanda interna y externa. La conclusión fundamental a la que llegamos es que, considerando todo el periodo de aplicación, la combinación de austeridad fiscal y reducciones salariales han provocado un aumento del desempleo en España.

\subsection{La evolución de los salarios y los costes laborales unitarios}

Los gráficos 6 y 7 recogen la evolución de los salarios nominales según los convenios firmados entre empresas y trabajadores $y$ registrados oficialmente, y según la Encuesta Trimestral del Costes Laborales realizada por el $\mathrm{INE}^{5}$. Indudablemente, en ambos casos se aprecia una sustancial caída de la tasa de crecimiento de los salarios nominales a partir de la crisis, y especialmente desde 2010. La tasa de crecimiento anual media de los salarios fue del 3,6\% entre 2001 y 2007 , pero desde entonces ha sido prácticamente igual a cero, y negativa (0,4\%) entre el tercer trimestre de 2012 y el último de 2014. Si nos fijamos en el salario real, utilizando como deflactor el índice armonizado de precios al consumo, se ha producido una pérdida de poder adquisitivo de los salarios del 8\% en relación al último trimestre de 2009.

$\mathrm{Y}$ si tenemos en cuenta los efectos composición del empleo, esta devaluación salarial es aún más evidente, porque la destrucción de puestos de trabajo se ha concentrado en empleados con menos nivel de cualificación y salarios más bajos (Puente y Galán, 2014).

Es cierto que la tasa de crecimiento de los salarios nominales aumentó durante el periodo 2008-2009, pero esto puede explicarse por

\footnotetext{
${ }^{5}$ La serie de los salarios pactados en los convenios hace referencia a los acuerdos entre las empresas y los sindicatos que se registran oficialmente, e incluyen tanto los nuevos convenios firmados en un año como las revisiones de convenios ya registrados anteriormente y con una duración plurianual. Sin embargo, aunque los salarios negociados son un buen punto de partida para ver en qué medida se están aplicando, por ejemplo, los posibles acuerdos de rentas pactados entre sindicatos y patronales, no recogen del todo la evolución de los salarios percibidos finalmente por los trabajadores, ya que hay que añadir después la evolución de otros complementos, la realización de horas extraordinarias o los acuerdos entre empresas y trabajadores al margen de los convenios sectoriales o nacionales, que pueden dar lugar a remuneraciones salariales por encima o por debajo de las inicialmente pactadas. Además, los cambios normativos que se han producido en el mercado de trabajo español han reducido sustancialmente en los dos últimos años el número de convenios registrados oficialmente y el porcentaje de trabajadores afectados. Por ello, un dato más ajustado de la evolución de los salarios medios del conjunto de trabajadores es el coste salarial por trabajador que se estima a través de la Encuesta Trimestral de Costes Laborales, elaborada por el Instituto Nacional de Estadística.
} 
diferentes factores recogidos en Pérez Infante (2013). En primer lugar, muchos convenios colectivos se habían firmado en los años anteriores, en un contexto de fuerte crecimiento económico y con un periodo medio de vigencia de más de tres años. Y aunque el empleo había empezado a decrecer ya en el sector de la construcción desde finales de 2007, la destrucción de empleo en la industria se produjo más tarde, por lo que las expectativas de los agentes sociales $-\mathrm{y}$ de las propias autoridadesno anticiparon plenamente la fuerte caída que se produjo a partir del tercer trimestre de 2008. En segundo lugar, el crecimiento acordado de los salarios nominales en los convenios registrados durante 2008 y 2009 sí fue decreciente, pero los salarios agregados subieron porque la mayoría de las pérdidas de empleo afectaron a trabajadores con contrato temporal, que en promedio reciben salarios más bajos que los trabajadores permanentes. Finalmente, influyó también la aplicación de las cláusulas de salvaguarda por la desviación al alza en la inflación de 2007, que fue dos puntos porcentuales a lo esperado en el momento de firmar la negociación colectiva para ese año. Esta compensación se pagó en 2008.

\section{Gráfico 6: Salarios nominales y Gráfico 7: Crecimiento anual de reales, crecimiento anual medio los salarios nominales}
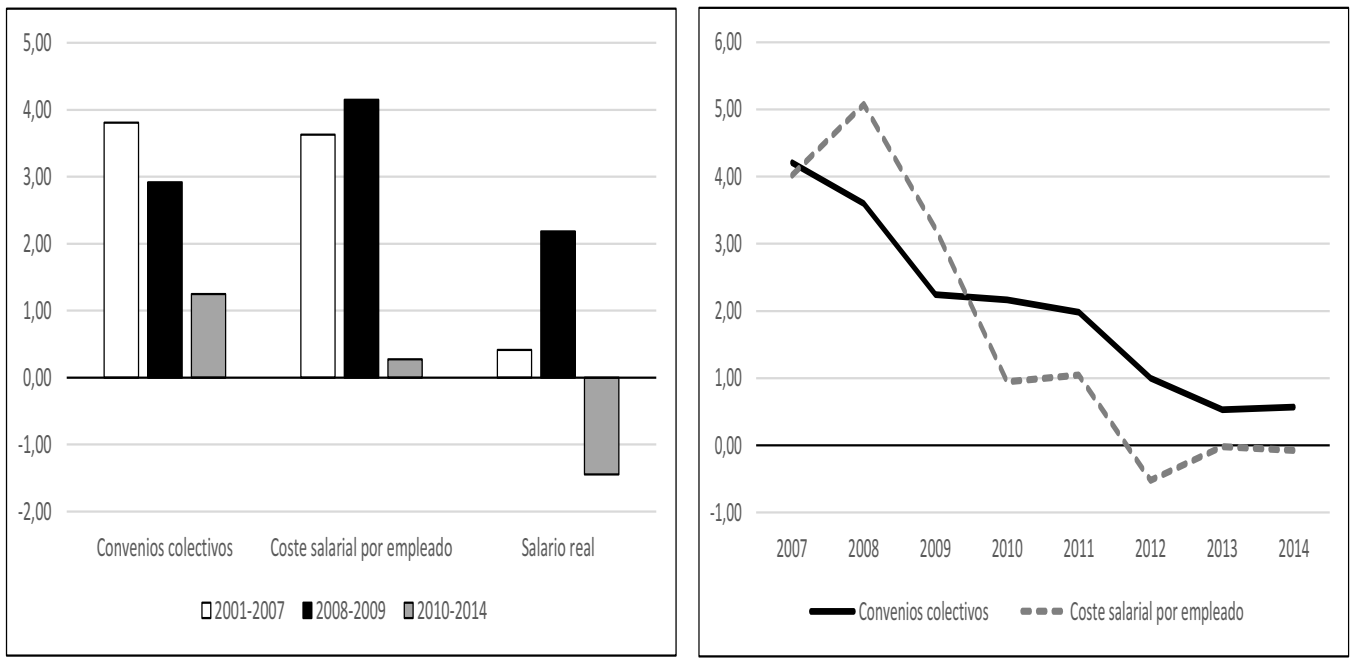

Fuente: Ministerio de Empleo y Seguridad Social, Instituto Nacional de Estadística y elaboración propia

Esta evolución de los salarios se debe, por un lado, a la propia crisis económica, ya que el aumento del paro ha debilitado la posición de 
los trabajadores en la negociación colectiva. En este sentido, los sindicatos y las organizaciones patronales firmaron en 2010 y 2012 dos Acuerdos para el Empleo y la Negociación Colectiva (ANEC) en los que se incluían recomendaciones para un crecimiento moderado de los salarios entre 2010 y 2014. Por ejemplo, el segundo de estos acuerdos establecía como límite superior un crecimiento salarial del 0,5\% en 2012 y el $0,6 \%$ en 2013. El crecimiento salarial de 2014 se condicionó a la evolución del PIB durante 2013, y finalmente quedó establecido por debajo del $0,6 \%{ }^{6}$.

Por otro lado, el comportamiento de los salarios en estos años también es la consecuencia deliberada de algunas medidas de política económica, y especialmente las que han afectado a la remuneración de los empleados públicos y la reforma laboral.

Los salarios de los trabajadores de las administraciones públicas se recortaron en 2010 y 2012, y se congelaron en 2011, 2013 y 2014. Aunque esto se presenta habitualmente como parte de los planes de consolidación fiscal, no hay que olvidar que el Pacto por el Euro firmado en 2011 ya destacaba el papel que pueden desempeñar los salarios públicos para "señalizar" el comportamiento que deberían observar las negociaciones salariales en el sector privado (Marzinotto y Turrini, 2014, analizan la relación entre los salarios en el sector público y en el sector privado).

Pero las decisiones de política económica con un impacto mayor sobre la negociación salarial han sido las reformas laborales aprobadas en 2010, 2011 y, sobre todo, 2012. Esta última incluía cambios muy sustanciales en tres áreas decisivas para la determinación de los salarios:

- Negociación colectiva: prioridad de los convenios de empresa en un amplio conjunto de materias, incluidas las salariales; ampliación de las posibilidades de inaplicación de las condiciones pactadas en el convenio vigente y para modificar unilateralmente por parte de la empresa las condiciones de trabajo que se hubieran pactado previamente por encima de los mínimos establecidos en el convenio colectivo; límite máximo de un año a la vigencia de un convenio tras su finalización.

- Costes y procedimientos de despido: redefinición de las causas económicas del despido; reducción de las indemnizaciones; y

\footnotetext{
${ }^{6}$ En junio de 2015 se firmó un III ANEC para los años 2015 a 2017. Los crecimientos salariales acordados son de hasta un $1 \%$ en 2015 y hasta un 1,5\% en 2016. El crecimiento salarial de 2017 se establecerá en función del crecimiento del PIB en 2016.
} 
supresión de la necesidad de autorización administrativa de los despidos colectivos.

- Modalidades de contratación: nuevo tipo de contrato indefinido para las empresas menores de 50 trabajadores con un período de prueba extendido a un año; flexibilización de las condiciones de los contratos de formación y aprendizaje y a tiempo parcial.

Izquierdo, Lacuesta y Puente (2013) tratan de aislar los efectos sobre los salarios de la propia situación de crisis económica -el aumento del paro- y de las medidas adoptadas por las autoridades, para lo que estiman una ecuación de salarios en los que éstos dependen de la situación del mercado de trabajo, la tasa de inflación y el crecimiento de la productividad. Observan que desde 2010 se aprecia un aumento del valor negativo de los residuos de esta ecuación, y especialmente a partir de finales de 2012, lo que se interpreta como un crecimiento de los salarios inferior al que se derivaría de sus determinantes básicos. Esto puede interpretarse como evidencia de que, efectivamente, las decisiones de política económica que hemos mencionado han tenido un efecto reductor de los salarios significativo.

Una consecuencia de esta fuerte moderación y posterior reducción de los salarios nominales es la reducción de los costes laborales unitarios (CLU) que se ha producido en España desde 2009, impulsada también por el mayor aumento de la productividad aparente del trabajo.

Desde el punto de vista del empleador, los costes laborales son más relevantes que los salarios por empleado, porque incluyen también otros conceptos como las cotizaciones sociales y los costes por despido, y porque compara estos costes con la productividad media de los empleados. La evolución de los CLU se puede calcular a través del cociente entre la remuneración por asalariado que ofrece la contabilidad nacional y la productividad real (el cociente entre el PIB a precios constantes y el número de ocupados).

Tomando el año 2000 como base, los costes laborales unitarios se incrementaron en España hasta el segundo trimestre de 2009, cuando se situaron un $16 \%$ por encima de la media de la eurozona. Desde entonces han registrado una reducción continuada y la diferencia con la eurozona ya era sólo del 3\% en 2014 (gráfico 8). Esta evolución es el resultado tanto de la reducción de los salarios nominales como de la evolución de la productividad aparente. En cualquier caso, ésta no se ha incrementado porque la producción haya crecido, sino por una reducción aún mayor del número de empleados y por la composición sectorial de los empleos destruidos. 
Conviene señalar que, si existe alguna relación entre los costes laborales y la demanda de trabajo por parte de las empresas, la magnitud relevante desde este punto de vista sería la de los costes unitarios reales (CLUR). Éstos comparan los salarios reales por trabajador con su productividad media, o producción real obtenida en promedio por cada trabajador. En este caso utilizamos el deflactor del PIB para obtener el salario real $^{7}$, porque estamos interesados en el punto de vista del empleador y no en la capacidad adquisitiva de los asalariados. Los CLUR tienen también una interpretación en clave distributiva: un aumento equivale a una elevación de la parte del valor añadido que es obtenida por los asalariados, mientras que una disminución implica -como ha ocurrido durante todo este periodo- un peso menor de las rentas salariales en la renta nacional ${ }^{8}$.

\section{Gráfico 8: Coste Laboral Unitario Nominal (2000Q4=100)}
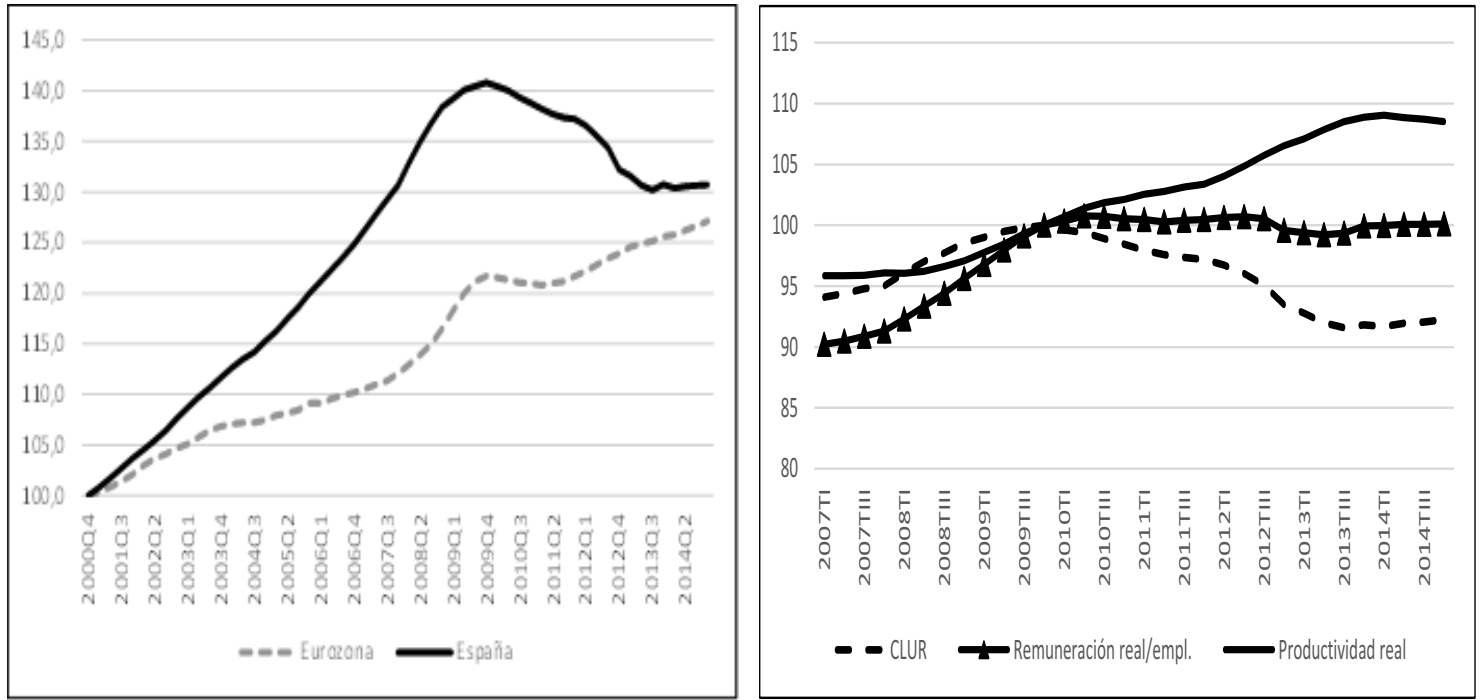

Fuente: Eurostat, INE y elaboración propia.

Como vemos en el gráfico 9, los CLUR han seguido una clara tendencia decreciente desde 2009, porque la remuneración real por empleado ha crecido de forma continua por debajo de la productividad

\footnotetext{
${ }^{7}$ Aproximado en este caso por el coste laboral.

8 Dada la tasa de asalarización.
} 
aparente (medida en términos reales). Como consecuencia, los CLUR fueron en 2014 un 8,5\% más bajos que en 2009.

De acuerdo con la teoría neoclásica de la demanda de trabajo, esto se debería haber traducido en niveles más altos de empleo, pero los datos de la evolución de los salarios y del paro y el empleo muestran claramente que la moderación salarial no ha provocado este efecto.

Al contrario, lo que observamos en el gráfico 10 es que, desde 2009, la moderación en el crecimiento de los salarios reales, y su reducción desde mediados de 2011 hasta finales de 2013, ha ido acompañada por un aumento de la tasa de desempleo. En el gráfico 11, por su parte, utilizamos los CLUR, para comparar esta evolución de los costes laborales con la productividad. Y vemos que a pesar de que los CLUR decrecieron entre 2010 y 2014 (el salario real no absorbió los fuertes aumentos de la productividad, sino que éstos se tradujeron en mayores márgenes de beneficio), esto no sirvió para estimular la contratación, y el empleo disminuyó.

\section{Gráfico 10: Costes Laborales reales y Gráfico 11: Coste Laboral Unitario desempleo Real y empleo}
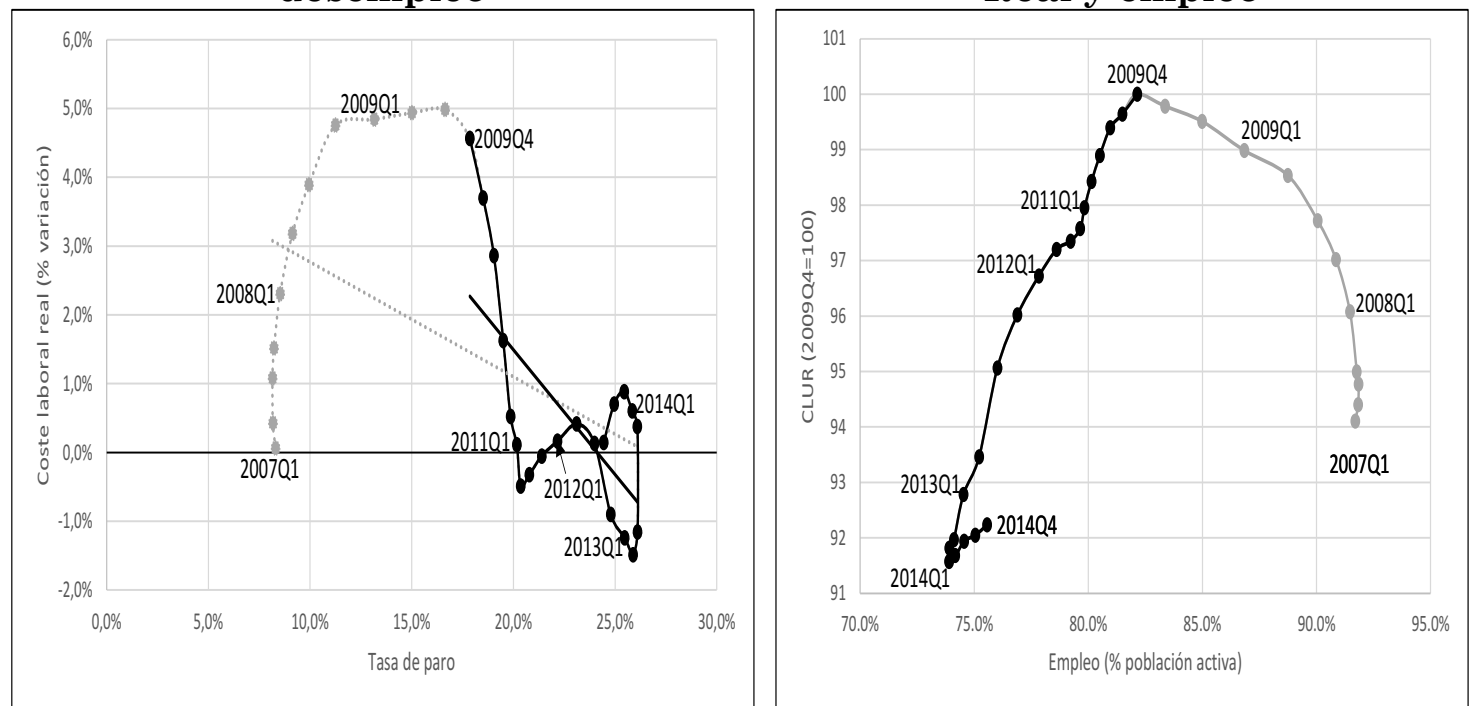

Fuente: INE y elaboración propia.

Nota: Cada punto corresponde a un trimestre, empezando en el primero de 2007 y terminando en el cuarto de 2014. La línea de puntos une el periodo 2007Q1-2009Q3, mientras que la línea continua recoge el periodo 2009Q4-2014Q4. En el gráfico 10 hay dos líneas de tendencia. La línea de puntos se refiere al periodo completo, mientras que la línea continua sólo a la segunda parte. 
De hecho, esto no debería sorprender. Primero, porque hemos visto en el apartado anterior que una gran proporción del aumento del desempleo está relacionada con el colapso de ramas concretas de actividad y que hay un porcentaje elevado de parados de larga duración con bajos niveles de cualificación. En estos casos, recortes salariales tienen poco efecto sobre el empleo. Y, segundo, porque la demanda de trabajo depende más de la demanda esperada para los bienes y servicios producidos por las empresas que con los costes laborales. Más aún, la reducción de los salarios tiene, desde el punto de vista macroeconómico, un fuerte efecto negativo sobre la demanda interna, que se suma a los efectos de la austeridad fiscal, mientras que ha tenido un efecto mucho más limitado sobre la demanda externa. Como vemos en la próxima sección, este último efecto no compensa la reducción de la demanda interna, y el resultado neto es un aumento del desempleo.

Tampoco hemos encontrado una relación sistemática entre el crecimiento de los salarios reales y el empleo a un nivel sectorial. Entre 2007 y 2011 se redujo el empleo en todos los sectores excepto en servicios financieros y de seguros, por un lado, y en los sectores relacionados con la administración pública y los servicios sociales, educación y sanidad, por otro. Sin embargo, el aumento del empleo en estas actividades no se debió a una reducción de sus salarios. Respecto al periodo de interés para nosotros (los años de la política de devaluación salarial, 2011-2014) la reducción de los salarios aparece asociada a aumentos del empleo en sectores concretos como el de las actividades inmobiliarias (los salarios nominales caen un 10,5\% y el empleo crece un 5\%). Pero, al mismo tiempo, la reducción salarial se asocia con pérdidas de empleo en otros como el comercio, transporte, hoteles y alimentación. Y la tendencia en la administración pública y los servicios sociales, educación y sanidad cambia respecto al periodo anterior, pero para pasar de un una tendencia creciente del empleo (10\%) y los salarios (2\%) en 2007-2011 a una caída tanto del empleo ($5 \%)$ como de los salarios (-1\%).

\subsection{La contribución negativa de la demanda interna al crecimiento}

En el gráfico 12 representamos la contribución al crecimiento de la demanda interna y de la demanda externa. Durante los años anteriores a la crisis se registraba una fuerte contribución positiva de la demanda interna, suficiente para compensar la contribución negativa de la demanda externa. Por el contrario, el sector exterior ha realizado una contribución positiva entre 2008 y 2013, pero muy insuficiente en 
relación con el importante deterioro de la contribución de la demanda interna, por lo que se ha registrado un crecimiento negativo del PIB.

El gráfico 13 representa, por su parte, la contribución al crecimiento de los distintos componentes de la demanda interna desde 2010, y nos permite mostrar el fuerte efecto restrictivo de la combinación de austeridad fiscal y devaluación salarial.

El efecto restrictivo de las políticas de austeridad fiscal se refleja en la contribución negativa del consumo público y la inversión pública, a lo que hay que añadir por supuesto el efecto multiplicador y la reducción directa de empleo público que hemos visto en la sección anterior. En términos reales, la suma de estos dos componentes de la demanda interna fue un $16,5 \%$ inferior en 2014 que en 2009 . Y en este mismo periodo su contribución negativa al crecimiento explica el $40 \%$ de la caída de la demanda interna.

De hecho, una parte importante del ajuste fiscal se deriva de reducciones de gasto público, sobre todo entre 2011 y 2013 . En este año, el gasto público total fue 25.000 millones menor que en 2010. En cuanto a los ingresos, aumentaron en 16.000 millones en 2010, pero desde entonces sólo se han elevado en 3.000 millones. A pesar de que las autoridades españolas han elevado los impuestos (sobre todo impuestos indirectos como el IVA, pero también impuestos sobre la renta) el aumento de los ingresos ha sido mucho menor que el previsto. Esto es consecuencia, al final, del fuerte impacto negativo de estas decisiones sobre la demanda agregada, que provocan un círculo vicioso de pérdidas acumulativas de producción e ingresos fiscales, junto a una explosión de la ratio entre la deuda pública y el PIB. Como consecuencia, no se alcanzan los objetivos de déficit, y se adoptan nuevas medidas de austeridad que incrementan el desempleo. El objetivo que se fijaron las autoridades en 2010, cuando se inició el proceso de consolidación fiscal, fue situar el déficit por debajo del $3 \%$ en 2013, pero el valor que finalmente se registró fue del $7,1 \%$ del PIB $(6,6 \%$ si no se tienen en cuenta las ayudas públicas a las instituciones financieras). Y en 2014 el déficit fue del 5,8\%. Este incumplimiento de los objetivos se debe principalmente a la caída en el PIB y la consiguiente reducción de los ingresos, y no al comportamiento del gasto público (Uxó, 2014). 
Gráfico 12: Contribución al crecimiento de la demanda interna y externa

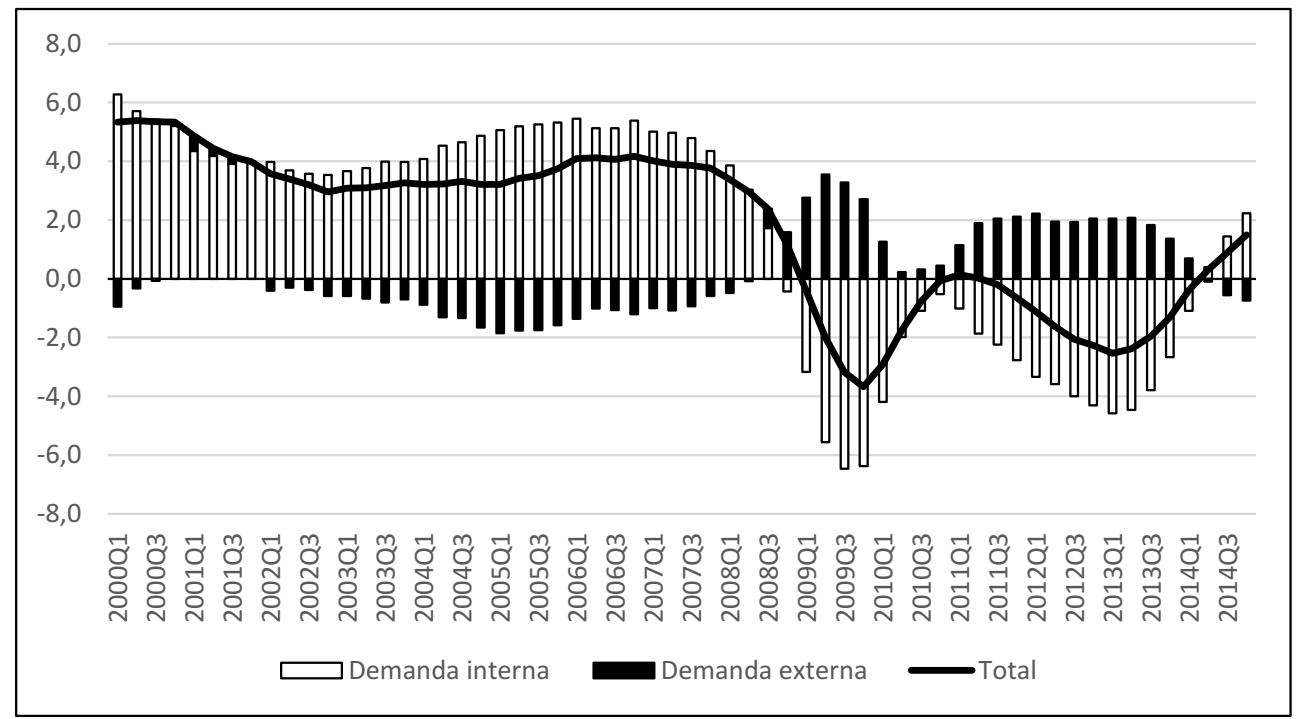

Fuente: INE y elaboración propia.

Gráfico 13: Contribución de los componentes de la demanda doméstica al crecimiento

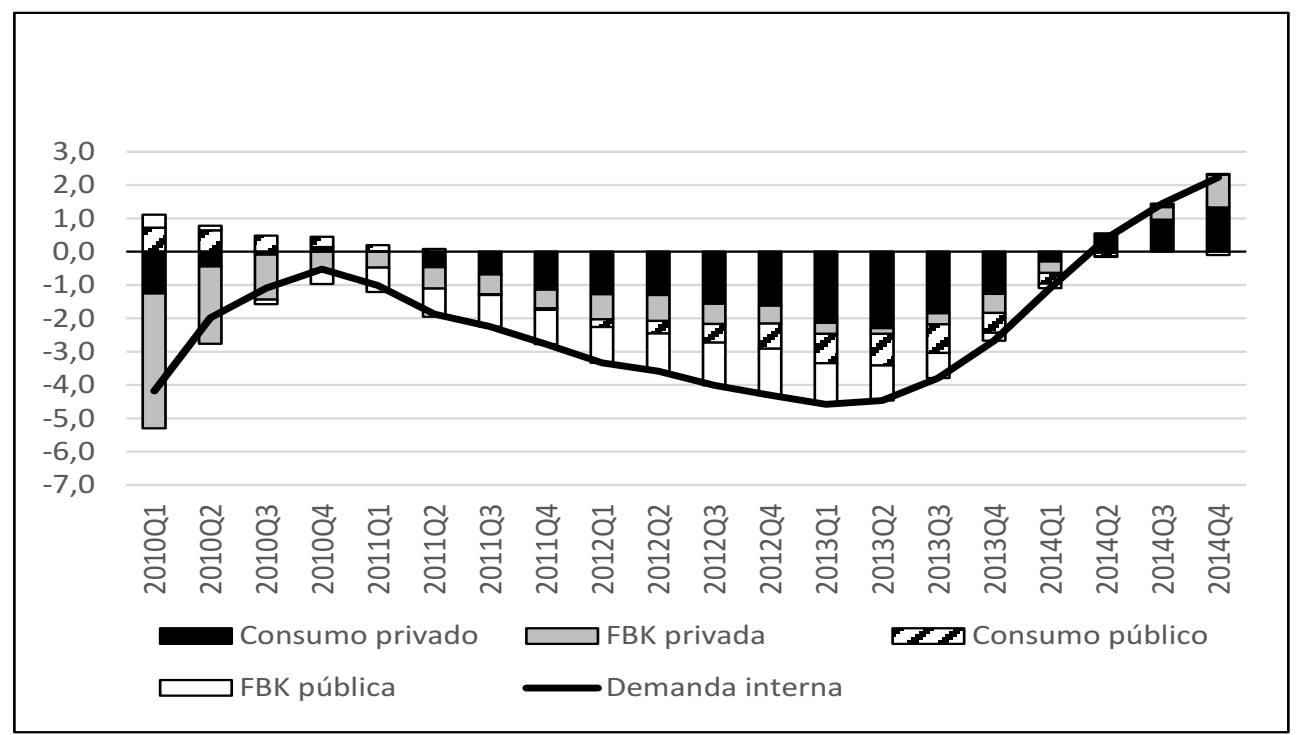

Fuente: INE y elaboración propia. 
También cabe destacar que la contribución de la demanda interna al crecimiento pasa a ser positiva a partir de 2014, y que esto coincide con un cambio en el signo de la política fiscal. En ese año, el gasto público total (neto de las ayudas a las instituciones financieras) crece por primera vez desde 2010, y el consumo público y la inversión pública dejan de tener una contribución negativa al crecimiento.

El consumo privado también registró una disminución en términos reales durante este periodo, y especialmente en 2012 y 2013 (gráfico 14): a finales de ese año, los hogares realizaban un gasto en consumo que era un 7\% inferior al de 2009 en términos reales, y en esos dos años la contribución media del consumo privado al crecimiento del PIB fue de -1,7\%. Esta caída es el resultado de una renta disponible de los hogares decreciente (gráfico 15), como consecuencia del comportamiento de los salarios que hemos descrito más arriba, ya que éstos son su principal componente. Y también pone de manifiesto uno de los efectos más negativos de la estrategia de devaluación interna: el aumento del peso real de la deuda.

\section{Gráfico 14: Consumo privado (2009Q4=100)}

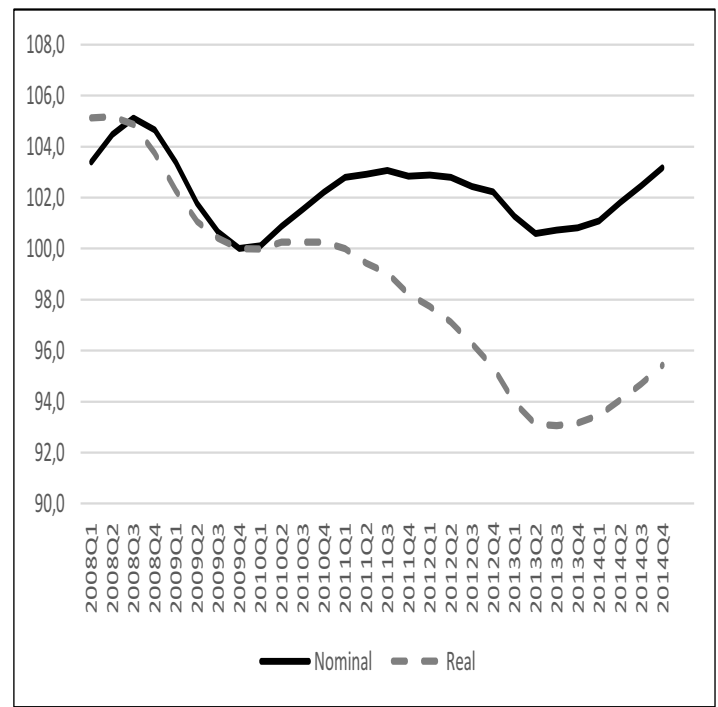

Fuente: Eurostat, INE y elaboración propia.
Gráfico 15: Renta disponible $(2009 \mathrm{Q} 4=100)$ y tasa de ahorro (\%)

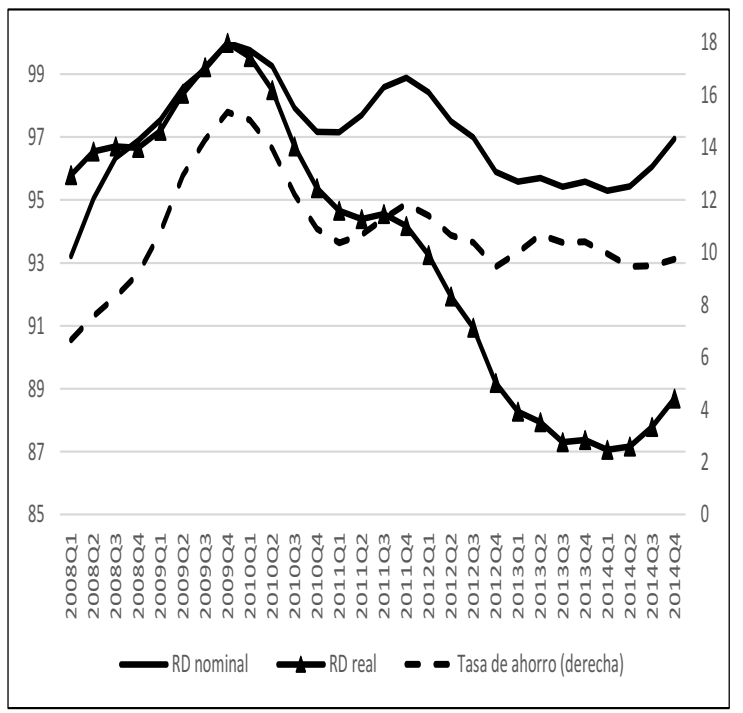


En un primer momento (2008-2009), el incremento de las transferencias públicas permitió que la renta nominal disponible de los hogares aumentase, a pesar de la caída del PIB. Sin embargo, el consumo nominal también se redujo en 2009, por el aumento de la tasa de ahorro (preventivo) que siguió al aumento de la incertidumbre. A partir de 2010 lo que observamos es que la destrucción de empleo, la consolidación fiscal y la devaluación salarial reducen la renta de los hogares. Y aunque inicialmente esto se tradujo en una reducción de la tasa de ahorro que permitió mantener el gasto nominal, esto deja de ser así a partir de 2011, y tanto el consumo nominal como el real estuvieron reduciéndose hasta 2013.

En cuanto a este comportamiento de la tasa de ahorro, Arce, Prades y Urtasum (2013) señalan que la propensión a ahorrar tiende a reducirse en los periodos en los que se producen reducciones muy importantes de la renta disponible, entre otras razones porque se alcanza un umbral mínimo de consumo de determinados bienes básicos. Cuando la renta se sitúa en valores inusualmente bajos, los hogares no pueden ajustar su gasto en la misma proporción, y la tasa de ahorro disminuye.

Esta reducción del ahorro es mucho más pronunciada en hogares con ingresos más bajos, pero otra diferencia importante se deriva de la existencia o no de deudas hipotecarias. Aquellos hogares con compromisos de pago derivados de hipotecas se ven obligados a realizar un "ahorro forzoso" para hacer frente al pago del servicio de la deuda, y el ajuste por la pérdida de renta recae en mayor medida en el consumo.

Efectivamente, los datos de consumo por hogares confirman que aquellos que tienen hipotecas pendientes incrementaron su tasa de ahorro en mayor medida entre 2006 y 2009, y que la redujeron menos que el resto posteriormente, cuando la renta disponible estaba disminuyendo. Es decir, que el endeudamiento tiene como consecuencia un mayor ajuste del consumo a partir de la crisis.

Esto refuerza la idea de que aquellas economías altamente endeudadas y que presentan déficits elevados por cuenta corriente, como España, corren el riesgo de enfrentarse al problema de la deflación de la deuda si tratan de equilibrar su sector exterior a través de la devaluación interna. Cuando la deuda es elevada, la reducción de los salarios se traduce en un peso relativo mayor del servicio de esa deuda, y esto reduce el consumo, que en España representa en torno al $60 \%$ de la demanda agregada.

El gráfico 16 muestra la evolución del incremento neto de la deuda y del servicio de la deuda en relación con la renta disponible de los hogares desde 2000. El primero tiene un efecto expansivo sobre la 
capacidad de gasto de los hogares, mientras que el segundo la reduce. Como vemos, los incrementos de deuda alcanzan su máximo a finales de 2006, antes de que la burbuja inmobiliaria estallase, y desde entonces ha estado reduciéndose, con la excepción de un breve repunte en 2011, asociado a algunos beneficios fiscales relacionados con la compra de nuevas viviendas. Respecto al servicio de la deuda, se ha mantenido entre el $10 \%$ y el $13 \%$ de la renta disponible de los hogares. Supone un "ahorro forzoso" para los hogares endeudados que además va a mantenerse en el tiempo, porque la mayor parte de esta deuda es a largo plazo (un $77 \%$ del total a mediados de 2014 ).

\section{Gráfico 16: Endeudamiento y servicio de la deuda (\% renta disponible de los hogares)}

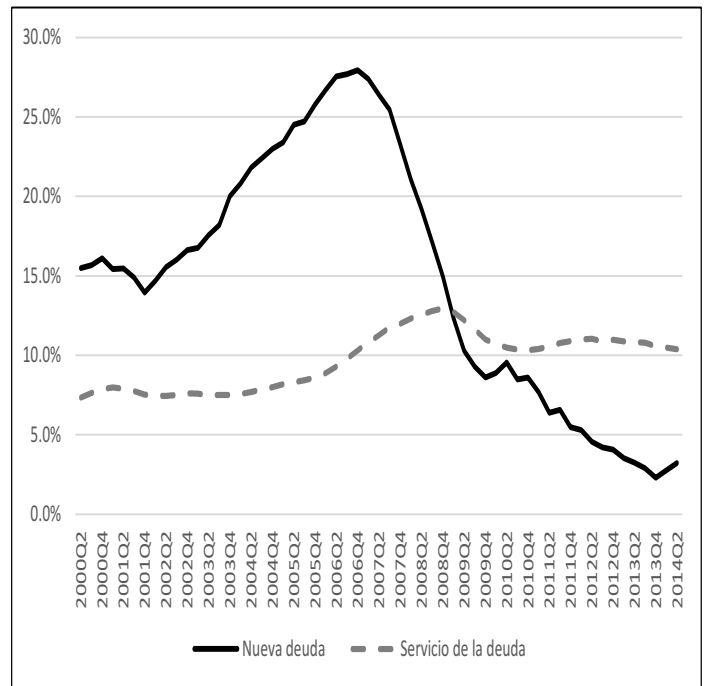

\section{Gráfico 17: Renta disponible de los hogares, endeudamiento y gasto (Millones $€$ )}

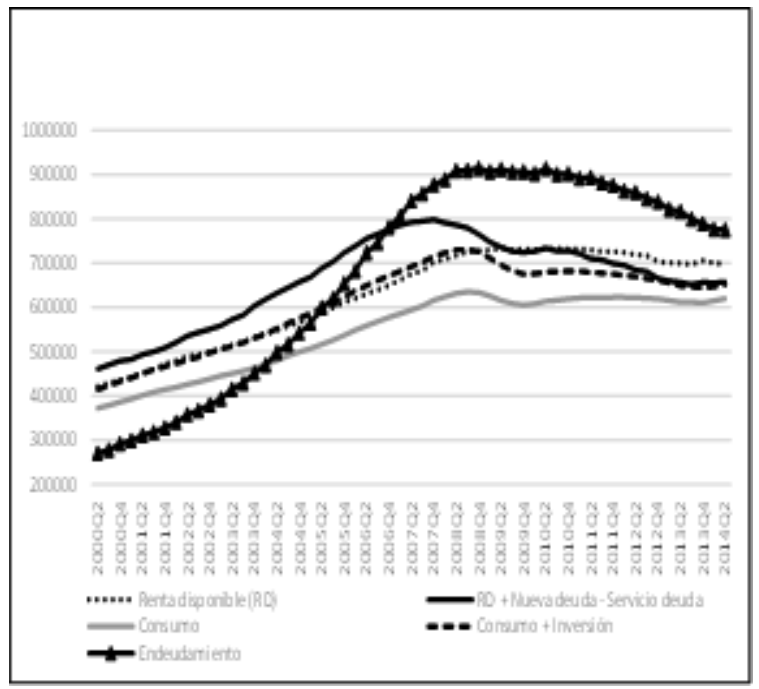

Fuente: Banco de España y elaboración propia

Nota: El nuevo endeudamiento es la diferencia entre la deuda bancaria total en el periodo $t+1$ y el periodo t, más la amortización del principal en ese periodo. El servicio de la deuda incluye el principal y los intereses. El gasto total es la suma del consumo final y la formación bruta de capital de los hogares.

En el gráfico 17, por su parte, observamos las consecuencias de este ahorro forzoso sobre el consumo de los hogares. La deuda total acumulada por los hogares alcanza su máximo a finales de $2007 \mathrm{y}$ comienza a disminuir desde entonces, por el proceso de desendeudamiento y el estancamiento del nuevo crédito. Sin embargo, 
el servicio de la deuda se mantiene relativamente elevado, como acabamos de decir, y el resultado es que la renta disponible neta, después de descontar los pagos asociados al endeudamiento pasado y el nuevo crédito, se ve reducida en torno a un 10\%. Desde mediados de 2012, los hogares españoles están realizando un gasto total (consumo más inversión) prácticamente una cantidad igual a la renta disponible que les queda después de los pagos del servicio de la deuda (intereses más principal).

El último componente de la demanda interna que nos queda por analizar es la formación bruta de capital privada. Teóricamente, la reducción del consumo que tiene lugar como consecuencia de la disminución de los salarios podría compensarse (aunque sólo parcialmente) por una mayor inversión por parte de las empresas, cuya rentabilidad estaba aumentando. Sin embargo, aunque la bajada de los costes laborales y el aumento de los márgenes de beneficios han dado lugar a un aumento de la renta disponible de las empresas durante estos años, podemos ver en el gráfico 13 que la inversión privada también ha tenido una contribución negativa al crecimiento desde 2010 hasta mediados de 2014. Esto se explica por la reducida utilización de la capacidad productiva instalada y la demanda estancada a la que se enfrentaban las empresas, y porque las empresas han destinado sus ingresos crecientes a reducir la deuda en mayor medida que a la inversión productiva.

En conclusión, la austeridad fiscal y la devaluación interna han tenido un efecto negativo sobre la demanda interna, contribuyendo al aumento del desempleo. $\mathrm{Y}$, como veremos a continuación, no han provocado el impulso expansivo sobre la demanda externa que teóricamente se esperaba para compensarlo.

\subsection{Devaluación interna y contribución de las exportaciones netas al crecimiento}

Las autoridades españolas y europeas confiaban en que los costes laborales decrecientes dieran lugar no sólo a un ajuste del sector exterior gracias a la mejora de la competitividad, sino también a un impulso de las exportaciones netas que fuese suficiente para recuperar el crecimiento y reducir el desempleo. Sin embargo, esto no ha ocurrido. Primero, porque la reducción de los salarios y los CLU se ha traducido en mucha menor medida en una mejora del tipo de cambio efectivo real (TCER) si se calcula utilizando precios de producción o de las exportaciones, que son medidas mucho más adecuadas de la competitividad que los CLU relativos (Wood, 2014). Segundo, porque la 
mejora de la balanza de pagos que se ha producido en España desde 2010 se explica principalmente por el colapso de las importaciones, que a su vez es el resultado de una demanda interna estancada, y no de un incremento de la competitividad precio, que en realidad ha sido mucho menor del esperado. Las exportaciones han crecido a un ritmo elevado, pero ya lo hicieron también en el periodo anterior a la crisis, como veremos.

\subsubsection{Cambios en la competitividad-precio}

Para medir la competitividad-precio utilizamos el tipo de cambio efectivo real frente a los 37 principales socios comerciales, calculado a partir de los costes laborales unitarios (TCER-CLU), el deflactor del PIB (TCER-DEF) o el deflactor de las exportaciones (TCER-EXP). Tomando 2000 como el año base, España registró una apreciación real hasta mediados de 2008, pero se ha producido una mejora de su competitividad desde entonces. Ahora bien, aunque la apreciación real se ha corregido en gran medida en términos de los costes laborales unitarios, la depreciación real ha sido mucho menor cuando se mide en términos del deflactor del PIB, y todavía más pequeña si se hace utilizando los precios relativos de las exportaciones. El TCER-CLU se ha reducido un 13\%, el TCER-DEF un 9\%, y el TCER-EXP sólo un 5\%. Lo que esto significa es que, a pesar de la fuerte devaluación salarial, la apreciación real en relación con el año 2000 todavía es de un 14\% cuando se utilizan el TCER-DEF o el TCER-EXP, y muestra claramente las limitaciones de la estrategia de devaluación interna como vía para mejorar la competitividad, por al menos tres razones.

En primer lugar, las diferencias entre la evolución de los tipos de cambio reales que se obtienen con los costes laborales y los que se obtienen con los precios finales se deben al diferente grado en que los cambios en los CLU se trasladan a los precios en un país y en sus competidores. Y aunque las tasas de inflación se han reducido, la devaluación salarial sólo se ha trasladado parcialmente a los precios en España: entre 2009 y 2014, los CLU se han reducido, pero los precios se han incrementado un $0,7 \%$ acumulado.

Esta diferencia se debe a que la tasa de crecimiento del deflactor del PIB no sólo depende de los CLU, sino también de los márgenes de beneficios y de los impuestos indirectos. Utilizando los datos de la contabilidad nacional (Uxó, Paúl y Febrero, 2014) podemos calcular las contribuciones de estos tres componentes a la tasa de inflación, que se muestran en el gráfico 18. Aunque la tasa de crecimiento anual media del deflactor del PIB ha sido muy moderada entre 2010 y 2014 (0,2\%), 
no refleja ni mucho menos toda la reducción de los CLU (-1,4\%) porque los márgenes de beneficios se incrementaron sustancialmente $(1,1 \%)$ desde que se iniciaron las políticas de devaluación interna. Además, se ha registrado una contribución positiva de los impuestos indirectos a los precios (0,5\% anual).

En segundo lugar, el resto de la zona euro también ha registrado tasas de crecimiento del deflactor del PIB claramente por debajo del $2 \%$, lo que pone de manifiesto también la dificultad de llevar a cabo un ajuste "asimétrico" de la competitividad, en el que el grueso del ajuste recae sobre las economías con problemas de déficit exterior. Un ajuste "simétrico" de la competitividad hubiera requerido que aquellos países que previamente registraron crecimientos muy débiles de los salarios y superávits por cuenta corriente, tuvieran ahora tasas de inflación por encima del $2 \%$ durante algún tiempo. Por el contrario, el mantenimiento de una política de contención salarial en el núcleo de la unión monetaria obliga a los países periféricos a implementar aún mayores recortes salariales, causando un sesgo deflacionario en el conjunto de la zona euro.

\section{Gráfico 18: Contribuciones de distintos factores al crecimiento del deflactor del PIB}

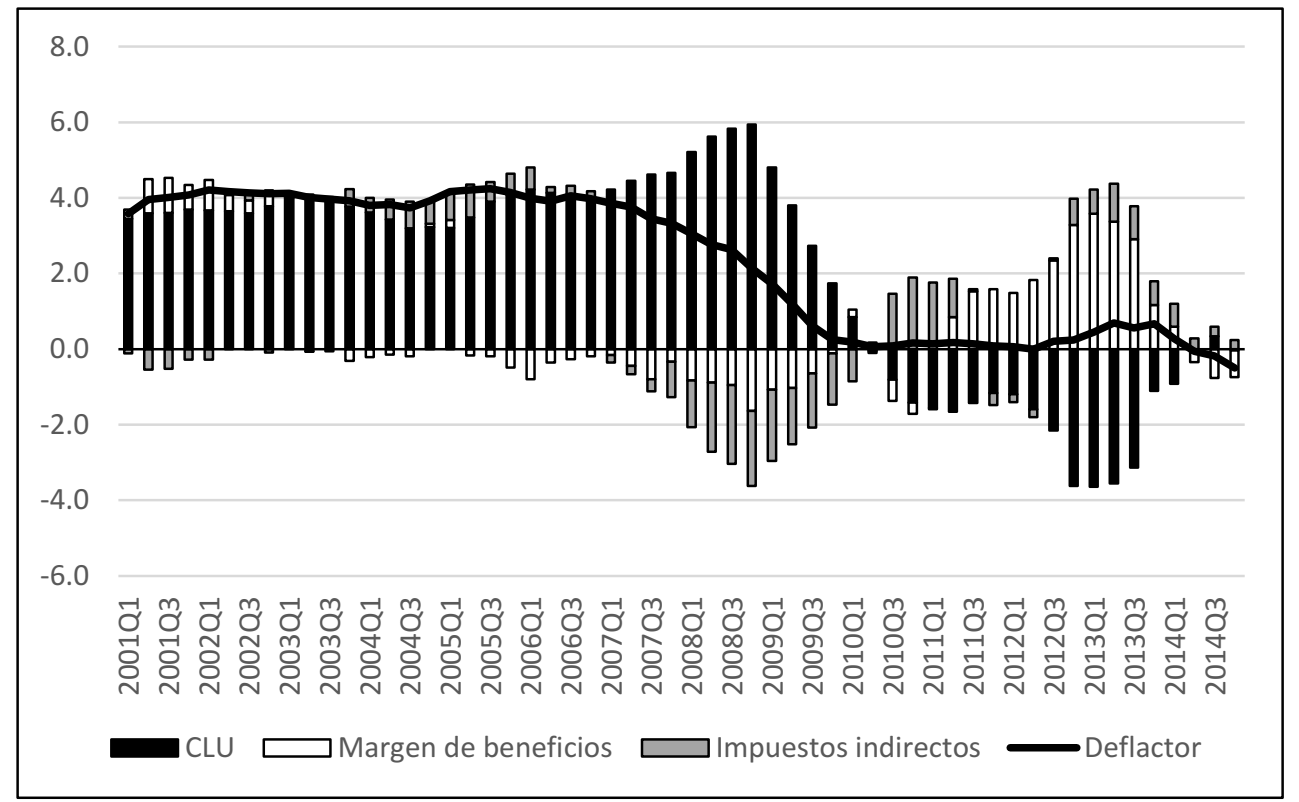

Fuente: INE y elaboración propia. 
$\mathrm{Y}$ un tercer factor que ha dificultado la reducción del tipo de cambio efectivo real es la apreciación nominal del euro registrada entre mediados de 2012 y principios de 2014. En gran medida, esto es también consecuencia de la naturaleza asimétrica del ajuste de las balanzas por cuenta corriente que se ha producido en los países del euro. El intento de generalizar una estrategia de crecimiento basado en las exportaciones en todos los países, y la continuidad de los superávits en los países centrales, hace que la zona euro en su conjunto pase a registrar superávit por cuenta corriente, provocándose la apreciación del euro. En el gráfico 19 distinguimos la parte de la evolución del TCER-EXP que se debe al cambio acumulado de los precios relativos de las exportaciones y la que se explica por la evolución del tipo de cambio nominal (en ambos casos, tomando como referencia el cuarto trimestres de 2009). Comprobamos que si bien la depreciación del euro contribuyó hasta la mitad de 2012 a reducir el TCER de España, su apreciación ha más que compensado desde entonces y hasta el fin de 2013 la mejora observada en los precios relativos de las exportaciones. Finalmente, esta tendencia se ha modificado en 2014, en gran medida por el cambio en la orientación de la política monetaria aplicada por el BCE.

\section{Gráfico 19: TCER con precios de exportación}

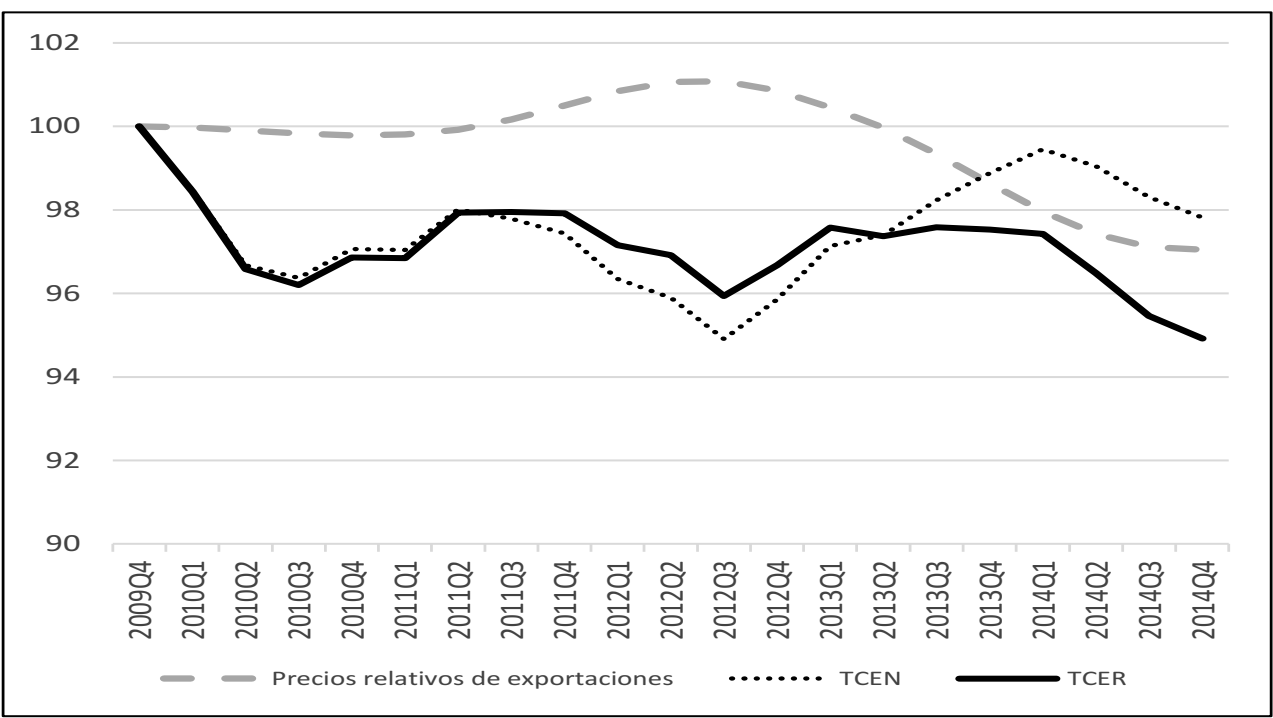

Fuente: INE y elaboración propia. 
Para concluir esta sección, conviene señalar que aunque el efecto sobre la competitividad-precio de la devaluación salarial ha sido más limitado de lo que la evolución de los salarios y los CLU podría indicar, sí se ha producido desde 2010 un aumento de la rentabilidad de las empresas en el sector de bienes comerciables, y que esto puede tener un impacto positivo en la localización de plantas productivas en España cuyo output es mayoritariamente exportado (Salas Fumás, 2014).

\subsubsection{Cambios en las exportaciones e importaciones}

España ha pasado de unas necesidades de financiación de aproximadamente un $10 \%$ del PIB en 2008 a tener capacidad de financiación en 2013 y 2014, principalmente por la mejora de la balanza de bienes y servicios. Esto ha permitido también que el sector exterior haya pasado de tener una fuerte contribución negativa al crecimiento a tenerla positiva entre 2010 y mediados de 2014. Aparentemente, estos dos hechos se pueden interpretar como una confirmación de que la estrategia de devaluación interna está alcanzando finalmente los resultados deseados, y que debería valorarse positivamente.

Sin embargo, vamos a ver que la reducción de las importaciones es el factor que más contribuye a explicar este cambio en el comportamiento del sector exterior, y a su vez el colapso de la demanda interna ha tenido más influencia que la evolución de los precios relativos (depreciación real). Por tanto, difícilmente puede ser considerado como una consecuencia "exitosa" de la devaluación interna.

El cuadro 10 recoge la evolución de las exportaciones e importaciones en términos reales, y su contribución al crecimiento económico, comparando los periodos 2010-2013 y 2000-2007, y recogiendo también lo ocurrido en 2014 (no consideramos los años 20082009, porque el comercio mundial se hundió). El crecimiento anual medio de las exportaciones se ha mantenido elevado en ambos periodos, y es mucho más notable el cambio en el comportamiento de las importaciones: mientras estaban creciendo antes de la crisis por encima del $7 \%$ anual, entre 2010 y 2013 se han reducido a un ritmo anual del $1,6 \%$.

Respecto al cambio de signo de la contribución al crecimiento del sector exterior, también se explica por la reducción de las importaciones, ya que la contribución positiva de las exportaciones es prácticamente la misma en los dos periodos. En cambio, la contribución anual de las importaciones al crecimiento ha pasado de $-2.0 \%$ a $0,5 \%$ (ver también el gráfico 20). 
Cuadro 10: Tasas de crecimiento de las exportaciones e importaciones y contribuciones al crecimiento del PIB

\begin{tabular}{ccccc}
\hline \multicolumn{2}{c}{ España } & $2000-$ & $2010-$ & 2014 \\
& & 2007 & 2013 & \\
\hline Contribución al & Demanda interna & 4,6 & $-2,9$ & 0,7 \\
crecimiento del PIB & Demanda externa & $-0,8$ & 1,6 & $-0,2$ \\
& Exportaciones & 1,2 & 1,1 & 1,4 \\
& Importaciones & $-2,0$ & 0,5 & $-1,5$ \\
Tasa de crecimiento & Exportaciones & 4,9 & 4,3 & 4,6 \\
real & Importaciones & 7,3 & $-1,6$ & 5,9 \\
\hline
\end{tabular}

Fuente: INE y elaboración propia.

Nota: Promedio de la tasa de crecimiento anual correspondiente a cada trimestre.

\section{Gráfico 20: Contribución de los componentes de la demanda doméstica al crecimiento}

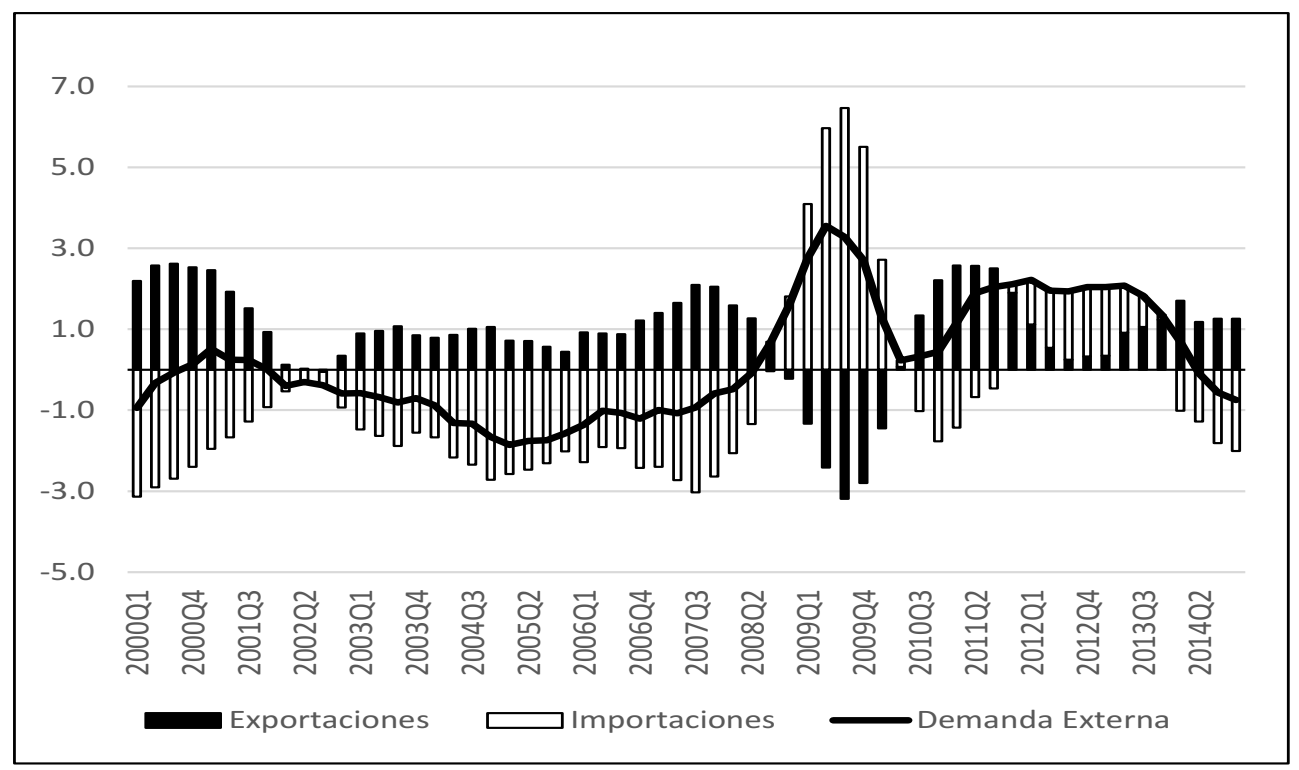

Fuente: INE y elaboración propia

Por último, Uxó, Paúl y Febrero (2014) muestran que este cambio en las exportaciones netas obedece sobre todo a la fuerte 
contracción de la demanda interna, y no a los efectos de una mejora en la competitividad. Para confirmarlo, estiman un modelo en el que la balanza de bienes y servicios depende: a) del cociente entre la demanda interna en la OCDE como un todo y la demanda interna en España; y b) del tipo de cambio efectivo real. Y obtienen que el signo de las elasticidades a largo plazo es el que se espera teóricamente: un incremento en la demanda relativa de la OCDE respecto a España mejora las exportaciones netas, mientras que una subida en el TCER (pérdida de competitividad) las empeora.

Utilizando estos resultados, el gráfico 21 compara los valores observados de las exportaciones netas (logaritmos) con dos valores hipotéticos. Primero, calculamos cuál habría sido la evolución de las exportaciones netas si la demanda interna hubiese crecido lo mismo en los últimos años en el conjunto de la OCDE y en España, de forma que el cociente entre ellas se hubiera mantenido en el mismo valor que en el cuarto trimestre de 2007 (línea continua). Alternativamente, con la línea de puntos representamos cuál habría sido la evolución de las exportaciones netas suponiendo que el tipo de cambio real no se hubiese modificado en ese mismo periodo. Es decir, en el primer caso eliminamos el efecto de las variaciones en la demanda interna, y en el segundo eliminamos el efecto de la depreciación real y tenemos en cuenta sólo la influencia de la demanda. Los resultados son muy claros: si no hubiera habido una caída tan pronunciada de la demanda interna, no se habría producido una corrección tan rápida del desequilibrio externo. En cambio, incluso manteniendo los precios relativos constantes la mejora de la demanda externa habría sido prácticamente la misma.

El comportamiento de las exportaciones netas en 2014 confirma lo que estamos señalando. Una vez que la economía española ha recuperado una tasa de crecimiento positiva, impulsada principalmente por una política monetaria del BCE más expansiva, la bajada de los precios del petróleo y una política fiscal más restrictiva, lo que ha provocado un cambio en la demanda interna, las importaciones han comenzado a incrementarse y la demanda externa está teniendo una contribución negativa al crecimiento.

Y todo lo que hemos visto en esta sección contradice la idea de que la devaluación interna puede impulsar una recuperación del crecimiento impulsada por las exportaciones. 


\section{Gráfico 21: Contribución de los componentes de la demanda doméstica al crecimiento}

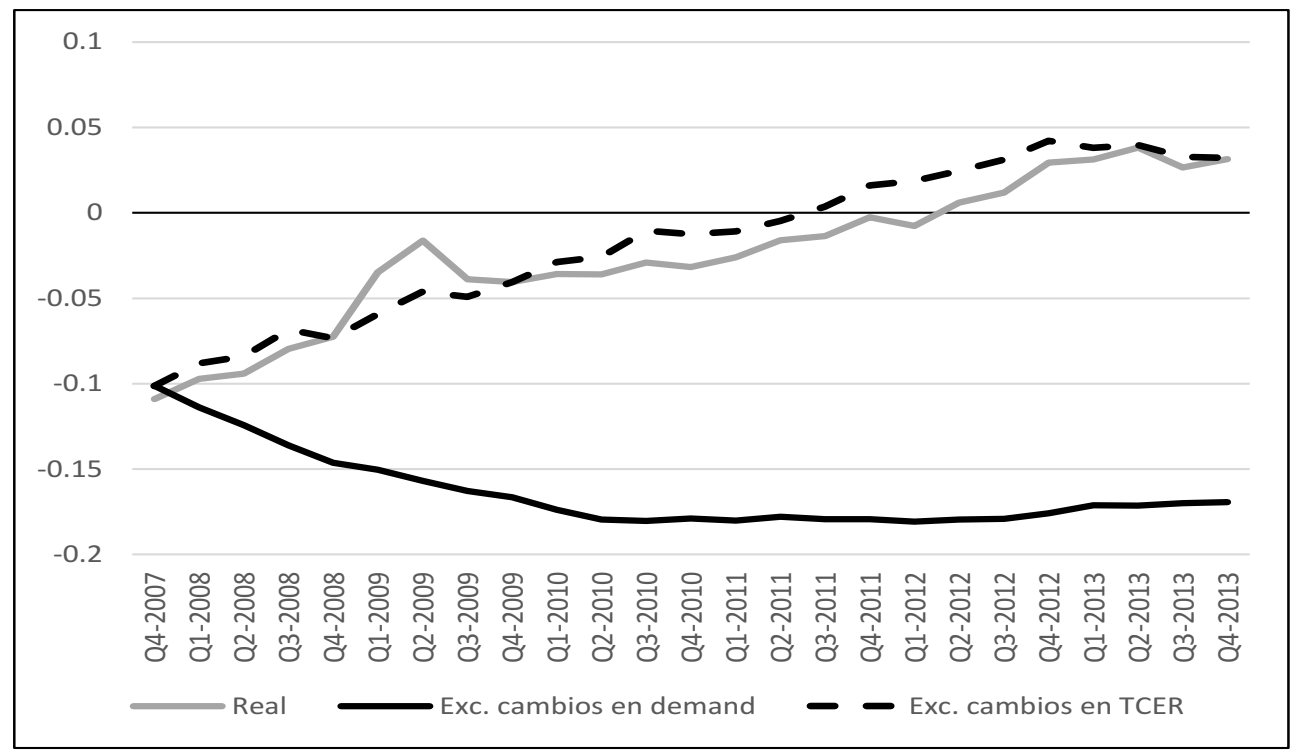

Fuente: OECD Economic Outlook, Uxó, Paúl y Febrero (2014) y elaboración propia.

\section{Conclusiones}

En este artículo hemos analizado los efectos de las reformas laborales recientes, y especialmente la de 2012, sobre los salarios y sobre el empleo, en el contexto de la estrategia global de política económica aplicada por las autoridades.

Una primera conclusión es que el aumento del desempleo por encima de la media europea que se ha producido durante la crisis está relacionado tanto con el tipo de shock experimentado (elevada especialización en el sector de la construcción y pinchazo de la burbuja) como con los efectos contractivos de la austeridad fiscal y la devaluación interna.

La reforma laboral persigue distintos objetivos, y nosotros nos hemos centrado aquí en sus efectos agregados sobre los salarios. Distintas medidas incluidas en esta reforma, y particularmente aquellas relacionadas con la negociación colectiva, han favorecido una bajada de los salarios nominales y los costes laborales unitarios. En este sentido, la reforma laboral es una parte fundamental de la estrategia de devaluación interna. Sin embargo, estos recortes salariales tienen poco 
efecto sobre el empleo cuando el problema se deriva del colapso de ramas concretas de actividad -construcción- que además genera un volumen elevado de parados de larga duración con bajos niveles de cualificación.

De hecho, lo que hemos encontrado es que, en contra de la visión convencional según la cual el empleo se incrementa con la reducción de los salarios (porque estimularía a las empresas a contratar más y porque incrementaría la competitividad y las exportaciones), en estos años se observa más bien una relación negativa entre la disminución de los costes laborales y el empleo. Esto se explicaría porque: a) los salarios decrecientes han tenido una contribución mucho menor que lo que suele afirmarse a la mejora de la competitividad-precio, principalmente porque los márgenes de beneficios y los impuestos indirectos se han elevado, y la bajada salarial sólo se ha trasladado parcialmente a los precios finales de los bienes exportados; b) el cambio en las exportaciones netas se explica sobre todo por el cambio en el comportamiento de las importaciones, y éstas a su vez disminuyen por el colapso en la demanda interna; y c) se ha producido una disminución de la renta disponible de los hogares y un aumento de la carga de la deuda en términos reales, debilitando el consumo final de los hogares, y esto se ha sumado a la contribución negativa al crecimiento de la demanda pública. En resumen, la caída de los salarios impulsada por la reforma laboral ha sido perjudicial para el empleo porque el efecto restrictivo sobre la demanda interna -que se suma al que se deriva de la austeridad fiscal- ha sido mayor que el impulso sobre la demanda externa.

La devaluación salarial sí ha permitido la recuperación de la rentabilidad de las empresas, y particularmente del sector comerciable, lo que puede favorecer la atracción de inversiones en ese sector y la reducción de la deuda empresarial. Esto ocurre, no obstante, con un fuerte impacto en términos distributivos.

Se ha producido un aumento sustancial del desempleo de larga duración, y las reformas laborales no han resuelto el problema de precariedad y dualización del mercado de trabajo. Cerca del 50\% de los 5,5 millones de desempleados que había en 2014 en España llevaban dos años o más sin trabajar o no lo habían hecho nunca, y el peso de los trabajadores con contrato temporal o a tiempo parcial sigue siendo muy elevado.

El aumento del empleo que se ha registrado en España desde mediados de 2014 no debería interpretarse como el resultado positivo de la estrategia de devaluación interna y bajadas salariales. Primero, porque para evaluar los efectos de estas políticas es necesario tener en 
cuenta sus consecuencias sociales y económicas durante todo el periodo en que se han aplicado, y entre 2011 han supuesto una pérdida muy sustancial de empleo y renta. Segundo, porque la recuperación actual encuentra su origen en el cambio en la política monetaria del BCE, la suavización del carácter restrictivo de la política fiscal y la caída de los precios de la energía. Y, tercero, porque muchos de los nuevos empleos son precarios y mal pagados.

\section{Bibliografia}

Alexiou, C. y Nellis, J.C. (2013), "Challenging the Raison d'etre of Internal Devaluation in the Context of the Greek Economy", Panoeconomicus, 60 (6), 813-836.

Arce, O., Prades, E. y Urtasun, A. (2013), "La evolución del ahorro y el consumo de los hogares españoles durante la crisis”, Boletín Económico, Banco de España, Septiembre, 27-35.

Banco Central Europeo (2012), "Euro Area labour markets and the crisis”, Monthly Bulletin, October, 69-80.

Dejuán, O. y Febrero, E. (2011), “The aftermath of a long decade of nil real interest rates (Spain, 1996-2008)", en Dejuán, O., Febrero, E. y Marcuzzo, M.C. (eds.), The first great recession of the 21st century. Competing explanations, Cheltenham, Edward Elgar, 222-246.

Febrero, E. y Bermejo, F. (2013), "Spain during the Great Recession", en Dejuán, O., Febrero, E. y Uxó, J. (eds.), Post-Keynesian Views of the Crisis and its Remedies, London y New York, Routledge, 265-293.

Fernández, C. e Izquierdo, M. (2013), "El ajuste de los mercados laborales europeos desde el inicio de la crisis”, Boletín Económico, Banco de España, Febrero, 45-55. 
Fondo Monetario Internacional (2010), Unemployment Dynamics during Recessions and Recoveries: Okun's Law and Beyond, World Economic Outlook, April.

Fondo Monetario Internacional (2012), World Economic Outlook, October.

Gobierno Español (2013), Plan Presupuestario 2013-2014. Disponible en:

http://ec.europa.eu/economy_finance/economic_governance/sgp/pdf/dbp/e s_2013-10-15_dbp_en.pdf

Izquierdo, M., Lacuesta, A. y Puente, S. (2013), “La reforma laboral de 2012: un primer análisis de algunos de sus efectos sobre el mercado de trabajo", Boletín Económico, Banco de España, Septiembre, 17-25.

Marzinotto, B. y Turrini, A. (2014), "The relationship between government and export sector wages and implications for competitiveness", Quarterly Report on the Euro Area, 13 (1), 27-34.

Myant, M. y Piasna, A. (2014), "Why have some countries become more unemployed than others? An investigation of changes in unemployment in EU member states since 2008", Working Paper 2014.07, Brussels, ETUI.

Pasinetti, L. L. (1973), "The notion of vertical integration in economic analysis", Metroeconomica, 25(1), 1-29.

Pérez Infante, J.I. (2013), “Crisis, reformas laborales y devaluación salarial”, X Jornadas de Economía Laboral, Universidad Autónoma de Madrid. Disponible en: https://www.uam.es/otros/jaeet13/comunicaciones/19_Reformas_laboral es/Perez_Infante.pdf 
Puente, S. y Galán, S. (2014), "Un análisis de los efectos composición sobre la evolución de los salarios”, Boletín Económico, Banco de España, Febrero, 25-28.

Salas-Fumás, V. (2014), "Las Sociedades No Financieras en España en la Crisis: Rentabilidad, Inversión y Endeudamiento", Mayo. Disponible en SSRN: $\underline{\text { http://ssrn.com/abstract=2436481 }}$

Timmer, M. et al. (2012), "The World Input-Output Database (WIOD): Contents, Sources and Methods", WIOD Background Document. Disponible en www.wiod.org.

Uxó, J. (2014), "La política de consolidación fiscal en España”, en A. Sánchez y J.A. Tomás Carpi (dirs.), Crisis y politica económica en España. Un análisis de la política económica actual, Thomson Reuters Aranzadi, 87-110.

Uxó, J., Paúl, J. y Febrero, E. (2011), "Current account imbalances in the monetary union and the great recession: causes and policies", Panoeconomicus, 2011-5, Special Issue, 571-592.

Uxó, J., Paúl, J. y Febrero, E. (2014), "Internal devaluation in the European periphery: the story of a failure", Working Paper 2014/2, Departmento de Análisis Económico y Finanzas, Universidad de Castilla - La Mancha.

Wood, R. (2014), "Eurozone Macroeconomic Framework: Reducing Internal and External Imbalances”, MPRA Paper Nº. 53569, Munich. 